\title{
Exact solutions for isothermal cyclic torsional loading of a circular SMA bar exploiting the shape memory effect
}

\author{
Enrico Radi ${ }^{1,2}$ \\ ${ }^{1}$ Dipartimento di Scienze e Metodi dell'Ingegneria, Università di Modena e Reggio Emilia, \\ Via G. Amendola 2, I-42122 Reggio Emilia, Italy.Email: enrico.radi@unimore.it \\ ${ }^{2}$ Centro Interdipartimentale “En\&Tech”, via G. Amendola, 2, I-42122 Reggio Emilia, Italy
}

\begin{abstract}
Two simple and fully analytical models are presented for a SMA bar or wire of circular cross section subjected to fully-reversed cyclic torsional loading, by taking into consideration the reorientation of the martensitic variants occurring during unloading and reverse loading. The process is assumed to take place at constant temperature between the start temperatures of the martensitic and austenitic transformations. In the first part of this work, the same shear modulus is taken both for Martensite and Austenite. In the second part, different elastic shear moduli are considered for the two phases. The volume fractions of both positive and negative twisted Martensite are assumed to evolve linearly with the shear stress. The bar is initially in a state of Austenite. As the applied torque is increased the martensitic transformation starts from the outer surface and then it extends inwards. If the maximum applied torque is large enough, then the complete Martensitic transformation takes place in the outer region of the cross section. During unloading and subsequent reverse loading the martensitic reorientation process may occur starting from the boundary between the fully martensitic outer region and the intermediate transforming region. Particular attention is focused on modeling the unloading and reverse loading processes. At each stage of the process, the radial distributions of shear stress and Martensite variant are calculated analytically. A closed form relation between the applied torque and the angle of twist is presented for the entire process in the case of equal shear moduli, and only for the loading and elastic unloading stages in the case of different shear moduli. The approach is then validated against analytical, numerical and experimental results available in the literature for the direct loading-unloading process. Application to the seismic response of dissipative systems based on SMA helical springs is also envisaged.
\end{abstract}

Keywords: Analytical solutions; Pure torsion; Circular cross section; Shape-memory alloy; Phase transformation; Martensite reorientation; Cyclic loading; Spring. 


\section{Introduction}

The problem of cyclic torsion of a shape memory alloy (SMA) bar of circular cross section is the fundamental basis for modeling the superelastic (SE) and the shape memory (SM) effects of SMA helical springs, which are finding ever more innovative applications in structural and civil engineering. In particular, SMA helical springs are ingeniously employed in passive protection and vibration control devices for civil structures subjected to severe seismic events (Dolce et al. 2000; Dolce and Cardone, 2001; DesRoche and Smith, 2004; Song et al., 2006; Speicher et al. 2009; Ozbulut et al., 2011, Torra et al., 2015, Heidari et al., 2016), due to their considerable energy dissipation and self-centering capabilities. The hysteretic behavior of tension-compression SMA springs is exploited specially in seismic isolators and dampers subject to alternating cyclic loading occurring during strong earthquakes. To this aim, it becomes important to enhance the hysteretic properties of SMA seismic devices by increasing their dissipation capability at each loading cycle, namely by exploiting the SM effect rather than SE behavior. Indeed, the cyclic reorientation process between positive and negative Martensite variants assures a larger amount of dissipated energy than SE behavior occurring with an intermediate austenitic transformation (Wilson and Wesolowsky, 2005). Due to the possibility of re-centering the device upon heating, SMA energy dissipative systems can thus efficiently replace frictional, viscous and elastic-plastic hysteretic devices in passive control and seismic retrofit of structural systems (Fang and Wang, 2020). In a SMA spring cyclically loaded at low temperatures, a large hysteresis loop similar to that exhibited by ductile metals is indeed observed, which is due to the reorientation process between martensitic variants rather than to phase transformation or dislocation glide mechanisms. Consequently, SMAs are able to display a much higher fatigue resistance than plastically deformed metals. For instance, superelastic Nitinol may endure cyclic strain amplitudes ranging from $4 \%$ to $12 \%$ for the number of cycles at which most other alloys would fail under cyclic strain amplitudes of $1 \%$ or less (Wilkes and Liaw, 2000). Also the stress-induced Martensite variants formed during Martensite reorientation process at low temperature are mobile and lead to a low buildup of defects and, hence, to a long fatigue life, see Figure 4 in Wilkes and Liaw (2000). Great fatigue resistance for martensitic Nitinol is observed typically in strain-based fatigue tests (Mahtabi et al., 2015). Modeling the hysteretic response of a SMA spring is however more complex than that of a steel spring, since it involves both processes of phase transformation and Martensite reorientation.

The problem of torsion of a circular SMA bar was investigated by Mirzaeifar et al. $(2010,2012)$ by using analytical solutions and finite element method. These authors examined the stress induced martensitic forward and backward transformation upon a loading-unloading cycle and proposed an integral relation between the torque and the angle of twist. They also provided a consistent review of 
previous works on the problem of torsion of SMA bars and tubes. Recently, Viet et al. (2019) worked out an analytical solution for the problem of SMA circular shaft subject to a SE loading-unloading cycle in torsion, which required the solution of a cubic equation for the shear stress in the phase transforming region during loading. These authors validated their model against numerical and finite element results and used it for simulating the SE behavior of SMA helical springs (Mirzaeifar et al., 2011; Heidari, et al. 2016; Viet et al., 2020). These analyses were performed at constant temperature $T$ higher than the finish temperature of the austenitic transformation $A_{f}$ in order to exploit the SE effect and thus the self-centering mechanism. In this case, the reverse austenitic transformation is completed during the unloading process, thus originating the SE effect. However, all these authors did not take into consideration reversed loading and the Martensite reorientation process occur during unloading and reversed loading, which is expected in a helical spring damping device under seismic loading. For exploiting the SM effect of SMA, and thus maximizing the energy dissipation during each loading cycle, the temperature $T$ must be lower than the start temperature of the austenitic transformation $A_{s}$. In this case, a negative shear stress necessarily arises in the bar cross section during the unloading process and it is still present after complete unloading as residual stress. If the negative shear stress is large enough, then it may trigger the martensitic reorientation process. Most of the analytical studies available in the literature for cycling loading of SMA circular bars are restricted to the SE behavior, namely to the range of temperature above $A_{s}$, and thus they can not be employed for simulating the SM effect occurring at temperature lower than $A_{s}$ only. Note that the temperature $A_{s}$ of various SMAs is usually higher than the room temperature, e. g. for NiTinol alloy ( $\mathrm{Ni}_{55} \mathrm{Ti}$ ) considered by Brinson (1993), Heidari et al. (2016) and Hashemi and Kadkhodaei (2018).

For complex cyclic loading, only finite element simulation can be found in the literature (Lim and McDowell, 1999; Chapman et al., 2011; Andani et al., 2013). A complete and detailed analytical study of the SM effect induced in a SMA circular bar subject to a torsional loading-unloading and reverse loading cycle at temperature lower than $A_{s}$ has never been performed. It necessarily requires the adoption of a constitutive SMA model that incorporates two opposite Martensite variants.

The present study aims to develop two exact analytical models, which allow for obtaining closedform solutions for the shear stress and Martensite distributions in a SMA bar with circular cross section during each step of the loading-unloading processes with opposite sign at constant temperature $T$ lower than $A_{s}$. The $1 \mathrm{D}$ constitutive models adopted here is derived from a phenomenological 3D model based on the von Mises effective stress and strain. It assumes that the Martensite volume fractions evolve as linear functions of the shear stress, in agreement with the 1D constitutive models for uniaxial loading proposed by Brinson (1993), Brinson and Huang (1996), Govindjee and Kasper (1997, 1999), Marfia and Rizzoni (2013), Fahimi, et al. (2019) for single and 
multi-variants Martensite. However, the reorientation of Martensite variants under torsional loading is taken into consideration here analytically for the first time. Recently, Radi (2021) performed an analytical investigation of the SM effect in a SMA beam under direct and reversed bending loading by adopting a 1D SMA constitutive model that incorporates two opposite Martensite variants. The reorientation process between multiple martensitic variants was also considered in a previous work on the response of SMA thick-walled cylinders under internal pressure (Radi, 2018).

The simplifying assumptions of linear phase transformation kinetic allow us to obtain a closed form solution for the shear stress and the distribution of Martensite volume fraction, also during the unloading and the reverse loading processes, which is one of the main challenges of the present study. In the proposed models, the variation of temperature due to the thermal-mechanical coupling of shape memory alloys is neglected and thus the isothermal solutions here obtained may be inaccurate for high loading-unloading rates. On the other side, due to the heat diffusion process a fully coupled thermal-mechanical model necessarily requires the adoption of a purely numerical procedure, e.g. as done by Mirzaeifar et al. (2012), Frost et al. (2016), and Mohammadzadeh et al. (2019). Heat generation during cyclic loading can actually increase the temperature above $A_{s}$, thus shifting the SMA response into the SE regime. Such an inconvenient can perhaps be avoided or at least delayed by using a liquid immersion cooling system, e.g. by placing the spring in a tube with a conducting fluid (Rao et al. 2015) or by using hollow spring as proposed by Spinella and Dragoni (2010) and Spinella et al. (2010). However, SE behavior at the end of seismic loading may also be advantageous for exploiting the self-centering property of the dissipating device.

The occurring of inhomogeneous deformation in the form of strain localization has been observed in thin walled SMA tube under combined tension-torsion loading and also under pure torsion (Reedlunn, 2020). A possible reason provided by the author is that it may be caused by the presence of residual bending stresses. Sun and Li (2002) indeed observed that the deformation mode of the tube changes gradually from localization and propagation under pure tension to homogeneous deformation under pure shear, and they did not detect distinct Martensite bands and macroscopic instability under pure torsion. Moreover, Peng et al. (2008), Chen et al. (2014), and Bronsteinet al. (2019) showed that the deformation-induced martensitic transformation substructures can be regarded as alternatively arranged parallel lamellas of Martensite and Austenite. In the case of pure torsion the thickness of the Martensite lamellas is fine, while in the case of pure tension the interlamellar spacing is much larger and the Martensite lamellas may grow to macroscopic bands, thus clarifying the different response of SMA under pure tension and under pure torsion. Therefore, in the present investigation an axially homogeneous deformation is assumed along the SMA rod. Also in the case of SMA springs a homogeneous deformation is expected, since the bending component of loading is secondary. 
By assuming homogeneous deformation, Heidari et al. (2016) studied the response of SMA spring and verified their numerical results by comparing them with experimental findings. The influence of geometrical parameters on dissipated energy were studied by Hashemi and Kadkhodaei (2018) for designing SMA springs with the purpose of achieving maximum dissipative performance, both under small and large deformations. Recently, Hashemi et al. (2019) employed an energy approach for fatigue analysis of SMA springs based on a constitutive model which takes into account ratedependency and geometric nonlinearities.

Despite the theoretical difficulties and the number of equations, analytic solutions have a lot of advantages. From them one can see clearly the role played by constitutive and geometrical parameters, and thus they allow understanding also more complex problems. Moreover, they provide a reliable evaluation of the residual stresses and strains in SMA springs after isothermal cyclic loading of alternating sign. Last but not least, exact solutions can be efficiently used also for validating the results of numerical procedures.

The paper is organized as follows: The 1D SMA constitutive model is presented in Section 2. The model is able to describe several phenomena occurring in the cyclic torsion of bars with circular cross section, such as phase transformations and Martensite variant reorientation. It keeps the essence of the approach used in Mirzaeifar et al. (2010, 2012) and Viet et al. (2019, 2020), but extends these studies by considering the Martensite reorientation process during unloading and reversed torsional loading. The closed form expressions of the shear stresses and Martensite fraction in all the different regions that arise within the cross section during loading-unloading and reversed loading are presented in detail in Sections 3 under the assumption of equal elastic shear moduli of the Austenite and Martensite phases. A closed-form relation between the applied torque and the angle of twist for the entire process is also presented in Section 3. The simplifying assumption of equal elastic shear moduli is then removed in Section 4, where different elastic shear moduli are considered for the two phases and the elastic shear modulus of the SMA material is assumed to vary with the Austenite and Martensite content according to the Reuss scheme for the elastic response of a composite. Note however that the definition of the Martensite shear modulus is still not clear, as pockets of retained Austenite still exist after the apparent martensitic transformation. By performing combined tensiletorsion tests on SMA tubes, Reddlunn et al. (2020) found indeed an almost constant value for the Austenite elastic modulus and observed a significant variation of the Martensite elastic modulus with the loading combination. In particular, in Fig 11 they observed almost equal values of the Austenite and Martensite shear moduli under pure torsion, thus validating the simplifying assumption made here in Section 3.

Results are then presented in Section 5 and the effects of the constitutive parameters on the radial 
distribution of shear stresses and Martensite volume fractions are therein discussed. Moreover, the analytical models are validated against similar analytical, numerical and experimental results available in the literature for the direct loading-unloading process. A summary of important results is given in Section 6. The proposed solution significantly improves upon existing work and extends the range of validity of the exact solution to two loading-unloading cycles of opposite sign.

\section{1D constitutive model for a SMA circular bar under torsion}

According to the linear elastic solution of the torsion problem for a circular cross section of outer radius $R$, the shear strain $\gamma$ is related to the angle of twist for unit length $\theta$ by

$$
\gamma=\theta r
$$

where $r$ denotes the radial distance within the circular cross section, for $0 \leq r \leq R$.

The applied torque $M$ is calculated by integrating the contribution of the shear stress $\tau$ on the cross section, namely

$$
M=2 \pi \int_{0}^{R} \tau r^{2} d r
$$

Let $\xi$ denotes the effective volume fraction of Martensite, which can be split as $\xi=\xi^{+}-\xi^{-}$, where $\xi^{+}$and $\xi^{-}$are the actual volume fraction of Martensite twisted in the positive (clockwise) and negative (anticlockwise) directions, respectively. Therefore, $\xi$ may range between -1 and 1 . The total volume fraction of Martensite is then $\xi_{\text {tot }}=\xi^{+}+\xi^{-}$. According to Govindjee and Kasper $(1997,1999)$ and Radi (2018), the 1D rate constitutive relationships between the shear stress $\tau$, the shear strain $\gamma$ in (2.1) and the effective volume fraction of Martensite twisted in the positive direction $\xi$ are

$$
r \dot{\theta}=\frac{\dot{\tau}}{G\left(\xi_{\text {tot }}\right)}+\gamma_{L} \dot{\xi}, \quad \dot{\xi}= \begin{cases}\frac{1+\xi_{0}}{\tau_{f}-\tau_{s}} & \text { for }\left|\tau_{s}\right| \leq|\tau| \leq\left|\tau_{f}\right|, \\ 0 & \text { otherwise, }\end{cases}
$$

where $G\left(\xi_{\text {tot }}\right)$ denotes the elastic shear modulus of the SMA material, which depends on the total Martensite volume fraction, $\gamma_{L}$ is the maximum transformation shear strain, namely $\gamma_{L}=\sqrt{3} \varepsilon_{L}$ being $\varepsilon_{L}$ the maximum inelastic strain attained under uniaxial loading when the solid is composed of fully oriented Martensite. Moreover, $\xi_{0}$ is the effective volume fraction Martensite at the beginning of the transformation, and $\tau_{s}=\sigma_{M s} / \sqrt{3}$ and $\tau_{f}=\sigma_{M f} / \sqrt{3}$ are the threshold shear stresses for the start and 
finish martensitic transformations, which depend on the temperature $T$ in agreement with the simplified phase diagram sketched in Fig. 1, namely

$$
\tau_{s}=\tau_{s}^{c r}+\frac{C_{\mathrm{M}}}{\sqrt{3}}\left(T-M_{s}\right), \quad \tau_{f}=\tau_{f}^{c r}+\frac{C_{\mathrm{M}}}{\sqrt{3}}\left(T-M_{s}\right),
$$

being $C_{\mathrm{M}}$ the slope of the martensitic transformation lines in the uniaxial stress-temperature phase diagram, $\sigma_{M s}$ and $\sigma_{M f}$ the uniaxial threshold stresses for the start and finish of the martensitic transformation (Brinson and Huang, 1996).

Let us assume that the SMA is fully austenitic initially, and thus $\xi=\xi_{\text {tot }}=\xi_{0}=0$. Then, the positive Martensite variant only is produced in the loading process according to the evolution law $(2.3)_{2}$ in the integrated form

$$
\xi=\xi^{+}= \begin{cases}0 & \text { for } \quad \tau \leq \tau_{s}, \\ \frac{\tau-\tau_{s}}{\tau_{f}-\tau_{s}} & \text { for } \quad \tau_{s} \leq \tau \leq \tau_{f}, \\ 1 & \text { for } \quad \tau \geq \tau_{f} .\end{cases}
$$

According to the linear evolution law $(2.3)_{2}$, the effective and total Martensite volume fractions during the unloading process are then given by (Govindjee and Kasper, 1997, 1999)

$$
\begin{gathered}
\xi=\xi^{+}-\xi^{-}= \begin{cases}\xi_{0} & \text { for } \tau \geq-\tau_{s} \\
\xi_{0}+\left(1+\xi_{0}\right) \frac{\tau+\tau_{s}}{\tau_{f}-\tau_{s}} & \text { for } \quad-\tau_{f} \leq \tau \leq-\tau_{s} \\
-1 & \text { for } \tau \leq-\tau_{f}\end{cases} \\
\xi_{\text {tot }}=\xi^{+}+\xi^{-}=\left\{\begin{array}{lll}
\xi_{0} & \text { for } \tau \geq-\tau_{s} \\
1-\left(1-\xi_{0}\right) \frac{\tau+\tau_{f}}{\tau_{f}-\tau_{s}} & \text { for }-\tau_{f} \leq \tau \leq-\tau_{s} \\
1 & \text { for } \tau \leq-\tau_{f}
\end{array}\right.
\end{gathered}
$$




\section{Cyclic torsional loading for equal shear moduli of the two phases}

For simplicity, in this section the same elastic shear modulus is considered both for Martensite and Austenite, equal to the mean value of the two moduli $G=\left(G_{\mathrm{A}}+G_{\mathrm{M}}\right) / 2$.

\subsection{Loading process}

The bar is initially in a state of Austenite and a torque $M$ is applied at the bar and gradually increased at constant room temperature $T$ between the start temperatures of the martensitic and austenitic transformations $\left(M_{s}<T<A_{s}\right)$, as depicted in Fig. 1. In the first pure elastic stage, the shear stress $\tau$ linearly increases with the torque and displays linear variation with the radius $r$ within the cross section, namely

$$
\tau=G \theta r=\frac{2 M}{\pi R^{4}} r
$$

according to (2.2) and (2.3). Therefore, the martensitic transformation starts at the outer radius $R$ when the shear stress reaches the threshold stress $\tau_{s}$, namely for $M=M_{s}$, where

$$
M_{s}=\frac{\pi R^{3}}{2} \tau_{s}
$$

Let

$$
\mu=\frac{M}{M_{s}}=\frac{4}{R^{3}} \int_{0}^{R} \frac{\tau}{\tau_{s}} r^{2} d r, \quad \alpha=\frac{G}{\tau_{s}} \theta R,
$$

denote the normalized applied torque and the normalized angle of twist, respectively, then during the pure elastic loading stage the following linear relation holds between them

$$
\mu=\alpha, \quad \text { for } 0 \leq \alpha \leq 1 .
$$

A further increase in the applied torque yields a progressive increase in the volume fraction of twisted Martensite, starting from the outer surface. Correspondingly, the front of the start of the Martensite transformation, defined by the radius

$$
r_{s}=\frac{\tau_{s}}{G \theta}=\frac{R}{\alpha}
$$

moves inward.

In the region undergoing martensitic transformation, the integration of the constitutive equations (2.3) from the start of martensitic transformation at radius $r$, which occurs for $\alpha=R / r$, for equal shear moduli $G$ of the two phases and for $\xi_{0}=0$, then yields

$$
\alpha \frac{r}{R}-1=\frac{\tau}{\tau_{s}}-1+\gamma_{L} \frac{G}{\tau_{s}} \xi, \quad \text { for } r_{s} \leq r \leq R .
$$


According to eqns (2.5), (3.5), and (3.6), in this stage of the loading process the shear stress and Martensite volume fraction within the cross section are given by

$$
\frac{\tau}{\tau_{s}}=\left\{\begin{array}{ll}
r / r_{s} & \text { for } \quad 0 \leq r \leq r_{s} \\
\frac{r / r_{s}+\delta}{1+\delta} & \text { for } \quad r_{s} \leq r \leq R
\end{array} \quad \xi= \begin{cases}0 & \text { for } \quad 0 \leq r \leq r_{s} \\
\frac{r / r_{s}-1}{(1+\delta) \beta} & \text { for } \quad r_{s} \leq r \leq R\end{cases}\right.
$$

respectively, where

$$
\delta=\frac{G \gamma_{L}}{\tau_{f}-\tau_{s}}, \quad \beta=\frac{\tau_{f}-\tau_{s}}{\tau_{s}} .
$$

A second front corresponding to the finish of the martensitic transformation originates when $\xi=1$ at $r=R$, namely for $\alpha=\alpha_{1}$ and $r_{s}=r_{1}$, where

$$
\alpha_{1}=\frac{R}{r_{1}}=1+\beta+\delta \beta, \quad r_{1}=\frac{R}{1+\beta+\delta \beta},
$$

according to (3.5) and (3.7) 2 , respectively. The relation between normalized applied torque and angle of twist during this stage then follows from the introduction of the stress field (3.7) in the balance condition $(3.3)_{1}$ as

$$
\mu=\frac{1}{1+\delta}\left(\alpha-\frac{\delta}{3 \alpha^{3}}+\frac{4}{3} \delta\right), \quad \text { for } 1 \leq \alpha \leq \alpha_{1} .
$$

When the shear stress reaches the threshold shear stress $\tau_{f}$ at $r=R$, the normalized applied torque (3.10) is

$$
\mu_{1}=1+\beta+\frac{\delta}{3(1+\delta)}\left(1-\frac{1}{\alpha_{1}^{3}}\right)
$$

A further increase in the applied torque makes the second front propagating inwards with radius $r_{f}$. Therefore, at the end of the loading process, the cross section is partitioned into three different regions: a purely austenitic inner region for $r \leq r_{s}$; an intermediate transforming region in a mixture of Austenite and Martensite for $r_{s} \leq r \leq r_{f}$ and an outer purely martensitic region for $r \geq r_{f}$.

Integration of eqn (2.3) in the outer fully martensitic region where $\xi=1$, starting from the finish of martensitic transformation at $r$, by using $(3.3)_{2}$, then gives

$$
\frac{\tau-\tau_{f}}{\tau_{s}}=\alpha \frac{r}{R}-\alpha_{1}, \quad \text { for } r_{f} \leq r \leq R,
$$

where

$$
r_{f}=\alpha_{1} r_{s}
$$

According to eqns (2.3), (2.5), (3.5), and (3.12) in the last stage of the loading process, the shear stress and Martensite volume fraction within the cross section are given by 


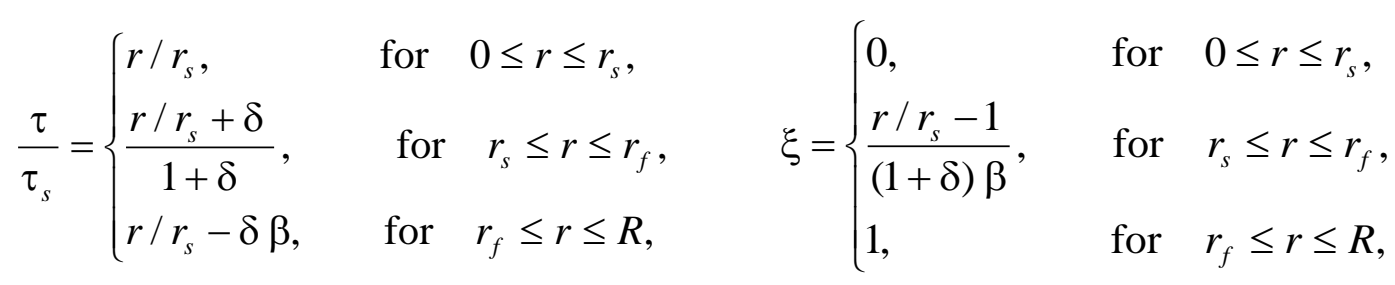

respectively. Therefore, both the shear stress and the Martensite volume fraction vary at most linearly with the radius $r$ within each region during the loading process.

The normalized applied torque $\mu$ then follows from the introduction of the stress field (3.14) in the balance condition (3.3) 1 as a nonlinear function of the normalized twisting angle $\alpha$

$$
\mu=\frac{\delta}{1+\delta} \frac{\alpha_{1}^{4}-1}{3 \alpha^{3}}+\alpha-\frac{4}{3} \delta \beta, \quad \text { for } \alpha_{1} \leq \alpha \leq \alpha_{\max }
$$

where $\alpha_{\max }=R / r_{s}$ denotes the normalized angle of twist at the end of the loading process, with $\alpha_{\max }$ $>\alpha_{1}$, being $\mu_{\max }$ the corresponding normalized torque, namely

$$
\mu_{\max }=\frac{\delta}{1+\delta} \frac{\alpha_{1}^{4}-1}{3 \alpha_{\max }^{3}}+\alpha_{\max }-\frac{4}{3} \delta \beta .
$$

Moreover, let $\tau_{\max }(r)$ and $\xi_{\max }(r)$ denote the radial distributions of shear stress and volume fraction of Martensite at the same stage, which are given in (3.14) for

$$
r_{s}=\frac{R}{\alpha_{\max }}, \quad r_{f}=\frac{\alpha_{1}}{\alpha_{\max }} R,
$$

according to (3.5) and (3.13).

\subsection{Unloading process}

As soon as the applied torque is decreased, an elastic unloading process takes place at every point in the cross section. During this process, the effective Martensite distribution $\xi_{\max }(r)$ as well as the radii $r_{s}$ and $r_{f}$ in (3.17) do not vary. The shear stress follows upon integration of the constitutive eqn (2.3) for equal shear moduli, using the definition $(3.3)_{2}$, as

$$
\frac{\tau}{\tau_{s}}=\frac{\tau_{\max }}{\tau_{s}}-\left(\alpha_{\max }-\alpha\right) \frac{r}{R}, \quad \text { for } 0 \leq r \leq R .
$$

If the temperature $T$ is lower than $A_{s}$ and the maximum applied torque $\mu_{\max }$ is large enough, then the elastic unloading of the bar ends when the shear stress (3.18) reaches the negative threshold stress $\tau_{s}$ at $r=r_{f}$, where $\tau_{\max }=\tau_{f}$, namely for $\alpha=\alpha_{2}$ where

$$
\alpha_{2}=\left(1-\frac{2+\beta}{\alpha_{1}}\right) \alpha_{\max }
$$

where eqns (3.17) have been used. The normalized torque-twist relation which holds during elastic 
unloading then follows from the introduction of the stress field (3.18) in the balance condition (3.3) 1 as

$$
\mu=\mu_{\max }+\alpha-\alpha_{\max }, \quad \text { for } \alpha_{2} \leq \alpha \leq \alpha_{\max },
$$

where $\mu_{\max }$ is given by (3.16). Let us denote with $\mu_{2}=\mu\left(\alpha_{2}\right)$ the minimum value of normalized torque during the elastic unloading stage, namely

$$
\mu_{2}=\mu_{\max }-(2+\beta) \frac{\alpha_{\max }}{\alpha_{1}}
$$

then, negative twisted Martensite takes place during the unloading process only if $\mu_{2}>0$, namely for $\alpha_{\max }>\alpha_{\lim }$ or equivalently for $\mu_{\max }>\mu_{\lim }$, where $\mu_{\lim }$ is the greater positive real root of the following quartic equation

$$
3\left(1-\frac{\alpha_{1}}{2+\beta}\right) \mu_{\lim }{ }^{4}+4 \delta \beta \mu_{\lim }^{3}-\frac{\delta(2+\beta)^{3}}{1+\delta}\left(\alpha_{1}-\frac{1}{\alpha_{1}^{3}}\right)=0
$$

Conversely, if $\mu_{\max }<\mu_{\text {lim }}$ then the unloading process is entirely elastic and the residual twist after complete unloading is given by $\alpha_{\mathrm{res}}=\mu_{\max }-\alpha_{\max }$, as it follows from (3.20) for $\mu=0$.

A further decrease in the applied torque, namely for $\mu<\mu_{2}$ and thus $\alpha<\alpha_{2}$, then triggers the reorientation of the positive Martensite variant into the negative one starting from the boundary between the fully martensitic outer region and the intermediate transforming region, namely at $r=r_{f}$ where $r_{f}$ is still given by $(3.17)_{2}$. From this circle, a new transforming region spreads out both inward and outward, where Martensite is reoriented, according to eqn (2.6). Let this new region be delimited by the inner radius $r^{\prime}$ and the outer radius $r^{\prime \prime}$, where $r_{s} \leq r^{\prime} \leq r_{f} \leq r^{\prime \prime} \leq R$. Then, the shear stress both at $r^{\prime}$ and $r^{\prime \prime}$ must coincide with the negative threshold stress $-\tau_{s}$. This condition is attained after elastic unloading starting from the shear stress distribution $\tau_{\max }(r)$ at the end of the loading process, namely

$$
\tau_{\max }\left(r^{\prime}\right)-\tau_{s}\left(\alpha_{\max }-\alpha\right) \frac{r^{\prime}}{R}=-\tau_{s}, \quad \tau_{\max }\left(r^{\prime \prime}\right)-\tau_{s}\left(\alpha_{\max }-\alpha\right) \frac{r^{\prime \prime}}{R}=-\tau_{s},
$$

for $r_{s} \leq r^{\prime} \leq r_{f} \leq r^{\prime \prime} \leq R$. By introducing in (3.23) the stress field $\tau_{\max }(r)$ at the end of the loading process given in (3.14) for $r_{s} \leq r \leq r_{f}$ and using eqn (3.5), one can find the radii $r^{\prime}$ and $r^{\prime \prime}$ as functions of the normalized angle of twist $\alpha$ :

$$
\frac{r^{\prime}}{R}=\frac{1+2 \delta}{\delta \alpha_{\max }-\alpha(1+\delta)}, \quad \frac{r^{\prime \prime}}{R}=\frac{\delta \beta-1}{\alpha},
$$

for $r_{s} \leq r^{\prime} \leq r_{f} \leq r^{\prime \prime} \leq R$. According to (3.24) 1 , a point at radius $r$ ranging between $r^{\prime}$ and $r_{f}$ starts experiencing Martensite reorientation when $\alpha=\alpha_{r}$, where $\alpha_{r}$ is given by

$$
\alpha_{r}=\frac{\delta \alpha_{\max }-(1+2 \delta) R / r}{1+\delta}, \quad \text { for } r^{\prime} \leq r \leq r_{f},
$$


and thus from (3.24) 1 and (3.25) it follows

$$
\alpha_{r}-\alpha=\frac{1+2 \delta}{1+\delta}\left(\frac{R}{r}-\frac{R}{r^{\prime}}\right), \quad \text { for } r^{\prime} \leq r \leq r_{f} .
$$

Let us consider a point at radius $r$ ranging between $r_{f}$ and $r^{\prime \prime}$ which also starts experiencing Martensite reorientation when $\alpha=\alpha_{r}$. Then, $\alpha_{r}$ must obey the following condition also

$$
\alpha_{r}=(\delta \beta-1) \frac{R}{r}, \quad \text { for } r_{f} \leq r \leq r^{\prime \prime},
$$

according to (3.24) 2 , and thus from (3.24) 2 and (3.27) it follows

$$
\alpha_{r}-\alpha=(\delta \beta-1)\left(\frac{R}{r}-\frac{R}{r^{\prime \prime}}\right), \quad \text { for } r_{f} \leq r \leq r^{\prime \prime} .
$$

During the Martensite reorientation process, the rate constitutive relations (2.3) for a material point at radius $r$, both for $r_{f} \leq r \leq r^{\prime \prime}$ and for $r_{f} \leq r \leq r^{\prime \prime}$ must hold in the following integrated form:

$$
\left(\alpha-\alpha_{r}\right) \frac{r}{R}=\frac{\tau}{\tau_{s}}+1+\delta \beta\left(\xi-\xi_{\max }\right)
$$

where $-\tau_{s}, \alpha_{r}$ and $\xi_{\max }$ are the shear stress, normalized twisting angle, and Martensite volume fraction at the beginning of Martensite reorientation process at radius $r$, respectively, whereas $\tau$, $\alpha$, and $\xi$ denote the same quantities evaluated during the Martensite reorientation process. Note that the initial Martensite volume fraction $\xi_{\max }$ is defined by (3.14) 2 for $\alpha=\alpha_{\max }$.

The introduction of (3.27) and (3.28) in eqn (3.29) then yields the following shear stress distribution in the new regions undergoing Martensite reorientation during the unloading process

$$
\begin{array}{ll}
\frac{\tau}{\tau_{s}}=-1-\frac{1+2 \delta}{(1+\delta)\left(1+\delta+\delta \xi_{\max }\right)}\left(\frac{r}{r^{\prime}}-1\right), & \text { for } r^{\prime} \leq r \leq r_{f}, \\
\frac{\tau}{\tau_{s}}=-1-\frac{\delta \beta-1}{1+2 \delta}\left(1-\frac{r}{r^{\prime \prime}}\right), & \text { for } r_{f} \leq r \leq r^{\prime \prime},
\end{array}
$$

where $r^{\prime}$ and $r^{\prime \prime}$ are given in (3.24) as functions of the normalized angle of twist.

The rest of the cross section, namely the inner part for $0 \leq r \leq r^{\prime}$ and the outer part for $r^{\prime \prime} \leq r \leq R$, undergoes only elastic unloading from $\alpha_{\max }$ to $\alpha$, and thus the shear stress within this part is given by

$$
\frac{\tau}{\tau_{s}}=\frac{\tau_{\max }}{\tau_{s}}-\left(\alpha_{\max }-\alpha\right) \frac{r}{R}, \quad \text { for } r^{\prime \prime} \leq r \leq R \text { and } 0 \leq r \leq r^{\prime}
$$

In particular, from (3.24) and (3.32) one obtain the shear stress as a function of $r$ and $r^{\prime}$ 


$$
\frac{\tau}{\tau_{s}}= \begin{cases}\frac{\delta}{1+\delta} \frac{r}{r_{s}}-\frac{1+2 \delta}{1+\delta} \frac{r}{r^{\prime}}, & \text { for } \quad 0 \leq r \leq r_{s}, \\ \frac{\delta}{1+\delta}-\frac{1+2 \delta}{1+\delta} \frac{r}{r^{\prime}}, & \text { for } \quad r_{s} \leq r \leq r^{\prime}, \\ \frac{\delta}{1+\delta} \frac{r}{r_{s}}-\frac{1+2 \delta}{1+\delta} \frac{r}{r^{\prime}}-\delta \beta, & \text { for } \quad r^{\prime \prime} \leq r \leq R .\end{cases}
$$

The distribution of effective Martensite within the regions undergoing Martensite reorientation then follows from the introduction of (3.30) and (3.31) into (2.6) for $\xi_{0}=\xi_{\max }$, namely

$$
\begin{array}{ll}
\xi=\xi_{\max }-\frac{\left(1+\xi_{0}\right)(1+2 \delta)}{\beta(1+\delta)\left(1+\delta+\delta \xi_{0}\right)}\left(\frac{r}{r^{\prime}}-1\right), & \text { for } r^{\prime} \leq r \leq r_{f}, \\
\xi=1-\frac{2(\delta \beta-1)}{\beta(1+2 \delta)}\left(1-\frac{r}{r^{\prime \prime}}\right), & \text { for } r_{f} \leq r \leq r^{\prime \prime} .
\end{array}
$$

The volume fraction of Martensite in the rest of the section does not change during the full unloading process, and thus it is still given by $\xi_{\max }$ in eqn $(3.14)_{2}$ for

During unloading, both the shear stress and the Martensite volume fraction vary linearly with the radius $r$ within the cross section, except for the regions undergoing Martensite reorientation delimited by the radii $r^{\prime}$ and $r_{f}$, where the distributions (3.30) and (3.34) are nonlinear functions of $r$, since the term $\xi_{\max }$ in the denominator is the linear function of $r$ provided in eqn (3.14)2. During the full unloading process, the total Martensite volume fraction in the cross section does not change, being $\xi^{+}$ $+\xi^{-}=\xi_{\max }$, since no backward transformation to Austenite is triggered at temperature $T$ lower than $A_{s}$. However, the effective Martensite volume fraction $\xi=\xi^{+}-\xi^{-}$is lower than $\xi_{\max }$ within the two new transforming regions. Indeed, the volume fraction $\xi^{-}$of Martensite has been transformed from the positive variant into the negative one during the last stage of the unloading process.

If the applied torque is decreased or applied with the opposite sign, the front of the start Martensite reorientation moves inward and outward, and it may reach the outer surface for $r^{\prime \prime}=R$, namely for $\alpha$ $=\alpha_{3}$, where

$$
\alpha_{3}=\delta \beta-1
$$

according to (3.24)2. The shear stress and Martensite distributions during this stage are still given by eqns (3.30)-(3.35).

The closed form expression of the normalized applied torque $\mu$ during this stage then follows from the introduction of the shear stress (3.30), (3.31), and (3.33) in the balance condition (3.3) 1 , namely

$$
\mu=\frac{4(1+2 \delta) \beta r_{s}}{\delta^{4} r^{\prime} R^{3}}\left(\eta r_{s}+\delta r^{\prime}\right)\left[\frac{\delta^{2}}{2}\left(r_{f}^{2}-r^{\prime 2}\right)-\delta \eta r_{s}\left(r_{f}-r^{\prime}\right)+\eta^{2} r_{s}^{2} \ln \frac{\delta r_{f}+\eta r_{s}}{\delta r^{\prime}+\eta r_{s}}\right]
$$




$$
\begin{aligned}
& +\frac{4 \delta}{3}\left[\frac{(\beta+2) r_{f}^{3}-2(1-\delta \beta) r^{\prime \prime}}{(1+2 \delta) R^{3}}-\beta\right]-\frac{(3+2 \delta) r^{\prime 3}+\delta r_{s}^{3}}{3(1+\delta) R^{3}}-\frac{4\left(r_{f}^{3}-r^{\prime 3}\right)}{3 R^{3}}\left[1+(1+2 \delta) \frac{\beta r_{s}}{\delta r^{\prime}}\right] \\
& +\frac{R}{1+\delta}\left(\frac{1+2 \delta}{r^{\prime}}-\frac{\delta}{r_{s}}\right)\left[\frac{r_{f}^{4}+2 \delta r^{\prime \prime}}{(1+2 \delta) R^{4}}-1\right], \quad \text { for } \alpha_{3} \leq \alpha \leq \alpha_{2},
\end{aligned}
$$

where

$$
\eta=\beta(1+\delta)^{2}-\delta
$$

The radii $r_{s}$ and $r_{f}$ are given in (3.17) and the radii $r^{\prime}$ and $r^{\prime \prime}$ are defined in (3.24) as functions of the normalized angle of twist $\alpha$.

For $\mu_{\max }>\mu_{\mathrm{lim}}$, the unloading process is not entirely elastic and the residual twist after complete unloading $\alpha_{\text {res }}$ is given by the greatest real solution of the equation $\mu\left(\alpha_{\text {res }}\right)=0$ satisfying $\alpha_{\text {res }} \leq \alpha_{2}$.

\subsection{Reversed loading-unloading cycle}

If the magnitude of the applied negative torque is increased after the complete unloading of the bar, then the outer region made of positive Martensite variant disappears as the radius $r^{\prime \prime}$ provided by (3.24) 2 turns out to be larger than $R$, namely for $\alpha<\alpha_{3}$. In the next stage of the process, the normalized angle of twist decreases until the negative threshold stress $-\tau_{f}$ for the finish of the reorientation process into negative Martensite variant is attained at the outer surface (at $r=R$ ). This condition is attained exactly for $\alpha=-\alpha_{1}$, according to (3.31) and (3.24)2.

The shear stress and Martensite distributions during this stage are given by eqns (3.30)-(3.35), by considering that $r^{\prime \prime}>R$. The corresponding torque-twist relation follows from the introduction of these shear stress fields in the balance condition $(3.3)_{1}$ as

$$
\begin{aligned}
& \mu=\frac{4(1+2 \delta) \beta r_{s}}{\delta^{4} r^{\prime} R^{3}}\left(\eta r_{s}+\delta r^{\prime}\right)\left[\frac{\delta^{2}}{2}\left(r_{f}^{2}-r^{\prime 2}\right)-\delta \eta r_{s}\left(r_{f}-r^{\prime}\right)+\eta^{2} r_{s}^{2} \ln \frac{\delta r_{f}+\eta r_{s}}{\delta r^{\prime}+\eta r_{s}}\right] \\
& -\frac{4 \delta(\beta+2)}{3(1+2 \delta)}\left(1-\frac{r_{f}^{3}}{R^{3}}\right)-\frac{(3+2 \delta) r^{\prime 3}+\delta r_{s}^{3}}{3(1+\delta) R^{3}}-\frac{4\left(r_{f}^{3}-r^{\prime 3}\right)}{3 R^{3}}\left[1+(1+2 \delta) \frac{\beta r_{s}}{\delta r^{\prime}}\right] \\
& +\frac{1}{1+\delta}\left(1-\frac{r_{f}^{4}}{R^{4}}\right)\left[\frac{\delta R}{(1+2 \delta) r_{s}}-\frac{R}{r^{\prime}}\right], \quad \text { for }-\alpha_{1} \leq \alpha \leq \alpha_{3},
\end{aligned}
$$

where $r^{\prime}$ is given in (3.24) $)_{1}$ as a function of $\alpha$.

If the magnitude of the negative torque is further increased, a region fully made of the negative Martensite variant originates at the outer part of the cross section and propagates inwards with radius $r_{-f}$ defined by the condition $\tau\left(r_{-f}\right)=-\tau_{f}$, namely 


$$
\frac{r_{-f}}{R}=-\frac{\alpha_{1}}{\alpha},
$$

according to (3.31) and (3.24)2. Correspondingly, the distributions of the shear stress and the effective Martensite volume fraction in the cross section are defined by eqns (3.32) and (3.14) for $0 \leq r \leq r^{\prime}$, by (3.30) and (3.34) for $r^{\prime} \leq r \leq r_{f}$, and by (3.31) and (3.35) for $r_{f} \leq r \leq r_{-f}$, respectively, where the radii $r^{\prime}$ and $r^{\prime \prime}$ are given in (3.24) as functions of $\alpha$. Moreover, integration of eqn (2.3) in the outer fully martensitic region where $\xi=-1$, starting from the finish of martensitic transformation at $r$, by using (3.3) 2 and (3.40), then yields the shear stress in the outer region

$$
\frac{\tau}{\tau_{s}}=-1-\beta+\alpha_{1}+\alpha \frac{r}{R}, \quad \text { for } r_{-f} \leq r \leq R .
$$

Note that for $\alpha=-\alpha_{\max }$ the shear stress distribution turns out to be equal but opposite to that corresponding to $\alpha=\alpha_{\max }$ and the effective Martensite distribution is given by $\xi=-\xi_{\max }$.

The torque-twist relation for $-\alpha_{\max }<\alpha<-\alpha_{1}$ follows from the introduction of the corresponding stress field in the balance condition (3.3) $)_{1}$ as

$$
\begin{aligned}
\mu= & \frac{4(1+2 \delta) \beta r_{s}}{\delta^{4} r^{\prime} R^{3}}\left(\eta r_{s}+\delta r^{\prime}\right)\left[\frac{\delta^{2}}{2}\left(r_{f}^{2}-r^{\prime 2}\right)-\delta \eta r_{s}\left(r_{f}-r^{\prime}\right)+\eta^{2} r_{s}^{2} \ln \frac{\delta r_{f}+\eta r_{s}}{\delta r^{\prime}+\eta r_{s}}\right] \\
& -\frac{(3+2 \delta) r^{\prime 3}+\delta r_{s}^{3}}{3(1+\delta) R^{3}}-\frac{4\left(r_{f}^{3}-r^{\prime 3}\right)}{3 R^{3}}\left[1+(1+2 \delta) \frac{\beta r_{s}}{\delta r^{\prime}}\right]+\frac{4 \delta}{3}\left(\beta-\beta \frac{r_{-f}^{3}}{R^{3}}-\frac{\beta+2}{1+2 \delta} \frac{r_{-f}^{3}-r_{f}^{3}}{R^{3}}\right) \\
& +\frac{1}{1+\delta}\left[1-\frac{r_{f}^{4}+2 \delta r_{-f}^{4}}{(1+2 \delta) R^{4}}\right]\left[\frac{\delta R}{r_{s}}-(1+2 \delta) \frac{R}{r^{\prime}}\right], \quad \text { for }-\alpha_{\max } \leq \alpha \leq-\alpha_{1},
\end{aligned}
$$

where $r^{\prime}$ and $r_{-f}$ are given in (3.24) 1 and (3.40) as functions of $\alpha$. Note from (3.42) that $\mu\left(-\alpha_{\max }\right)=$ $-\mu_{\max }$. If the negative torque is released from $-\mu_{\max }$ to 0 and then applied with the positive sign for completing a loading cycle, then the response of the section is similar to that observed for the unloading process, except that $\mu$ and $\alpha$ have the opposite sign. 


\section{Cyclic torsional loading for different shear moduli of the two phases}

In this section, different shear moduli $G_{\mathrm{A}}$ and $G_{\mathrm{M}}$ are considered for Austenite and Martensite and the elastic shear modulus of the SMA material is assumed as a function of the total volume fraction of Martensite according to the Reuss bond for the elastic response of a composite of two phases, namely

$$
G\left(\xi_{\text {tot }}\right)=\left(\frac{1-\xi_{\text {tot }}}{G_{\mathrm{A}}}+\frac{\xi_{\text {tot }}}{G_{\mathrm{M}}}\right)^{-1}
$$

\subsection{Loading process}

In the initial state of Austenite, during the pure elastic loading stage the following linear relation holds between the shear stress and the angle of twist

$$
\frac{\tau}{\tau_{s}}=\alpha \frac{r}{R}, \quad \text { for } 0 \leq \alpha \leq 1
$$

where

$$
\alpha=\frac{G_{\mathrm{A}}}{\tau_{s}} \theta R
$$

Correspondingly, the following linear relation holds between the normalized torque and angle of twist

$$
\mu=\alpha, \quad \text { for } 0 \leq \alpha \leq 1 .
$$

As the applied torque is increased, the front of the start of the Martensite transformation, defined by the radius

$$
r_{s}=\frac{R}{\alpha}
$$

takes place at the outer surface and then moves inward.

In the transforming region the following rate relation holds according to eqns (2.3) and (4.1)

$$
r \dot{\theta}=\left(\frac{1-\xi}{G_{\mathrm{A}}}+\frac{\xi}{G_{\mathrm{M}}}+\frac{\gamma_{L}}{\tau_{f}-\tau_{s}}\right) \dot{\tau}, \quad \text { for } r_{s} \leq r \leq R .
$$

By using eqn (2.5), eqn (4.6) can be integrated from the start of the martensitic transformation at $r$, namely

$$
G_{\mathrm{A}} \theta r-\tau_{s}=\int_{\tau_{s}}^{\tau}\left(1+\delta+\frac{\tau-\tau_{s}}{\beta \tau_{s}} \Gamma\right) d \tau, \quad \text { for } r_{s} \leq r \leq R,
$$

where

$$
\delta=\frac{\gamma_{L} G_{\mathrm{A}}}{\tau_{f}-\tau_{s}}, \quad \Gamma=\frac{G_{\mathrm{A}}-G_{\mathrm{M}}}{G_{\mathrm{M}}} .
$$


The definition of parameter $\delta$ in (4.8), which holds within the present section, is slightly different from that given in (3.8) and used in Section 3 for equal shear moduli of the two phases.

By calculating the definite integral in (4.7), one obtains the following quadratic equation for the shear stress $\tau$

$$
\left(1+\delta-\frac{\Gamma}{\beta}\right)\left(\frac{\tau}{\tau_{s}}-1\right)+\frac{\Gamma}{2 \beta}\left(\frac{\tau^{2}}{\tau_{s}^{2}}-1\right)=\alpha \frac{r}{R}-1, \quad \text { for } r_{s} \leq r \leq R .
$$

Therefore, the shear stress within the cross section is given by

$$
\frac{\tau}{\tau_{s}}= \begin{cases}r / r_{s}, & \text { for } \quad 0 \leq r \leq r_{s}, \\ 1+(1+\delta) \frac{\beta}{\Gamma}\left[\sqrt{1+\frac{2 \Gamma}{\beta(1+\delta)^{2}}\left(\alpha \frac{r}{R}-1\right)}-1\right], & \text { for } \quad r_{s} \leq r \leq R,\end{cases}
$$

and the Martensite volume fraction follows from (2.5) and (4.10) as

$$
\xi= \begin{cases}0, & \text { for } \quad 0 \leq r \leq r_{s}, \\ \frac{1+\delta}{\Gamma}\left[\sqrt{1+\frac{2 \Gamma}{\beta(1+\delta)^{2}}\left(\alpha \frac{r}{R}-1\right)}-1\right], & \text { for } \quad r_{s} \leq r \leq R .\end{cases}
$$

Note that both the shear stress and the volume fraction of Martensite display a weak nonlinear variation with the radius $r$ in the transforming region, which become linear as $\Gamma \rightarrow 0$, namely for equal elastic moduli of the two phases.

A second front corresponding to the finish of the martensitic transformation originates when $\xi=1$ at $r=R$. Correspondingly, the normalized angle of twist and the front of start martensitic transformation are given by

$$
\alpha_{1}=1+\left(\frac{\Gamma}{2}+1+\delta\right) \beta, \quad r_{1}=\frac{R}{\alpha_{1}},
$$

according to (4.11) and (4.5), respectively. The relation between normalized applied torque and angle of twist during this stage then follows from the introduction of the shear stress (4.10) in the balance condition (3.3) 1 as

$$
\begin{array}{rlrl}
\mu(\alpha)= & \frac{4 \beta(1+\delta)}{105 \alpha^{3} \Gamma}\left[5-3 B+2 B^{2}-2 B^{3}+\sqrt{1+2 \frac{\alpha-1}{B+2}}\left(30 \alpha^{3}+3 \alpha^{2} B-2 \alpha B^{2}+2 B^{3}\right)\right] \\
& +\frac{4}{3}\left(1-\frac{B+2}{1+\delta}-\frac{1}{4 \alpha^{3}}\right), & \text { for } 1 \leq \alpha \leq \alpha_{1},
\end{array}
$$

where

$$
B=\frac{\beta}{\Gamma}(1+\delta)^{2}-2 .
$$


When the shear stress reaches the threshold shear stress $\tau_{f}$ at $r=R$, the normalized applied torque is $\mu_{1}=\mu\left(\alpha_{1}\right)$.

A further increase in the applied torque makes the second front propagating inwards with radius $r_{f}$. Therefore, at the end of the loading process, the cross section is partitioned into an inner purely austenitic inner region for $r \leq r_{s}$; an intermediate transforming region for $r_{s} \leq r \leq r_{f}$ and an outer purely martensitic region for $r \geq r_{f}$.

Integration of eqn (2.3) for the outer fully martensitic region, where $\xi=1$ and $G(1)=G_{\mathrm{M}}$, starting from the finish of martensitic transformation at $r$, by using (4.3), then gives

$$
\frac{\tau-\tau_{f}}{\tau_{s}}=\frac{1}{1+\Gamma}\left(\alpha \frac{r}{R}-\alpha_{1}\right), \quad \text { for } r_{f} \leq r \leq R,
$$

where

$$
r_{f}=\frac{\alpha_{1}}{\alpha} R
$$

is the radius of the front of finish martensitic transformation, namely where $\tau=\tau_{f}$. During the last stage of the loading process, the shear stress and Martensite volume fraction within the cross section are

$$
\frac{\tau}{\tau_{s}}= \begin{cases}\frac{\alpha r}{R}, & \text { for } 0 \leq r \leq r_{s}, \\ 1+(1+\delta) \frac{\beta}{\Gamma}\left[\sqrt{1+\frac{2 \Gamma(\alpha r-R)}{\beta(1+\delta)^{2} R}}-1\right], & \text { for } \quad r_{s} \leq r \leq r_{f}, \\ 1+\beta+\frac{1}{1+\Gamma}\left(\alpha \frac{r}{R}-\alpha_{1}\right), & \text { for } \quad r_{f} \leq r \leq R,\end{cases}
$$

and

$$
\xi= \begin{cases}0, & \text { for } 0 \leq r \leq r_{s}, \\ \frac{1+\delta}{\Gamma}\left[\sqrt{1+\frac{2 \Gamma(\alpha r-R)}{\beta(1+\delta)^{2} R}}-1\right], & \text { for } r_{s} \leq r \leq r_{f}, \\ 1, & \text { for } r_{f} \leq r \leq R,\end{cases}
$$

respectively, as they follow from (4.10), (4.11), and (4.15).

Therefore, during the loading process both the shear stress and the effective Martensite volume fraction vary linearly with the radius $r$ within the inner austenitic and the outer martensitic regions, and display nonlinear variation in the intermediate region undergoing martensitic transformation.

The normalized applied torque $\mu$ then follows from the introduction of the stress field (4.17) in the balance condition (3.3) 1 and as a nonlinear function of the normalized twisting angle $\alpha$ 


$$
\mu(\alpha)=\frac{C}{\alpha^{3}}+\frac{\alpha}{1+\Gamma}+\frac{4}{3}\left(1+\beta-\frac{\alpha_{1}}{1+\Gamma}\right), \quad \text { for } \alpha_{1} \leq \alpha \leq \alpha_{\max }
$$

where

$$
\begin{aligned}
C= & \frac{4 \beta(1+\delta)}{105 \Gamma}\left[\frac{\left(B+2 \alpha_{1}\right)^{3 / 2}}{\sqrt{B+2}}\left(15 \alpha_{1}^{2}-6 \alpha_{1} B+2 B^{2}\right)-35\left(\alpha_{1}^{3}-1\right)-(B+2)\left(15-6 B+2 B^{2}\right)\right] \\
& +\frac{\alpha_{1}^{4}}{3(1+\Gamma)}-\frac{1+4 \beta \alpha_{1}^{3}}{3},
\end{aligned}
$$

and $\alpha_{\max }=R / r_{s}$ denotes the normalized angle of twist at the end of the loading process, being $\mu_{\max }=$ $\mu\left(\alpha_{\max }\right)$ the corresponding normalized torque.

Moreover, let $\tau_{\max }$ and $\xi_{\max }$ denote the radial distributions of shear stress and volume fraction of Martensite at the same stage, which are given in (4.17) and (4.18) for $\alpha=\alpha_{\max }$, respectively.

\subsection{Unloading process}

During the elastic unloading the Martensite distribution as well as the radii $r_{s}$ and $r_{f}$ do not change. For $\alpha=\alpha_{\max }>\alpha_{1}$, they are given by (4.5) and (4.16), respectively. Then, by integrating the rate constitutive equation (2.3) for $\xi=\xi_{\max }(r)$, and thus for $\dot{\xi}=0$, using (4.1), the shear stress during elastic unloading follows as

$$
\frac{\tau}{\tau_{s}}=\frac{\tau_{\max }}{\tau_{s}}-\frac{\alpha_{\max }-\alpha}{1+\Gamma \xi_{\max }(r)} \frac{r}{R}, \quad \text { for } 0 \leq r \leq R,
$$

and the corresponding normalized applied torque is

$$
\mu=\mu_{\max }-\left(\alpha_{\max }-\alpha\right) \frac{4}{R^{4}} \int_{0}^{R} \frac{r^{3}}{1+\Gamma \xi_{\max }} d r,
$$

according to (3.3) $)_{1}$ and (4.21). The substitution $t=1+\Gamma \xi_{\max }$ into (4.22), by using (4.18), then provides

$$
\mu=\mu_{\max }-\left(\alpha_{\max }-\alpha\right) m,
$$

where

$$
m=\frac{1}{\alpha_{\max }^{4}}\left\{1+\frac{\alpha_{\max }^{4}-\alpha_{1}^{4}}{1+\Gamma}+\frac{\beta^{4}}{2 \Gamma^{4}} \int_{1}^{1+\Gamma}\left[(t+\delta)^{2}-(1+\delta)^{2}+2 \frac{\Gamma}{\beta}\right]^{3}\left(1+\frac{\delta}{t}\right) d t\right\},
$$

and the integral in (4.24) has been calculated in Appendix A.

If the temperature $T$ is lower than $A_{s}$ and the maximum applied torque $\mu_{\max }$ is large enough, then the elastic unloading of the bar ends when the shear stress (4.21) reaches the negative threshold $-\tau_{s}$ at $r$ $=r_{f}$, where $\xi_{\max }=1$ and $\tau_{\max }=\tau_{f}$, namely when $\alpha=\alpha_{2}$ and $\mu=\mu_{2}$, being 


$$
\alpha_{2}=\alpha_{\max }-(1+\Gamma)(2+\beta) \frac{\alpha_{\max }}{\alpha_{1}}, \quad \quad \mu_{2}=\mu_{\max }-(1+\Gamma)(2+\beta) \frac{\alpha_{\max }}{\alpha_{1}} m
$$

Therefore, the Martensite reorientation process can take place during elastic unloading only if $\mu_{2}>0$, namely if $\alpha_{\max }>\alpha_{\lim }$, where $\alpha_{\lim }$ is given by the largest positive real root of the following quartic equation

$$
\left(\frac{1}{1+\Gamma}-\frac{2+\beta}{\alpha_{1}}\right) \alpha_{\lim }^{4}+\frac{4}{3}\left(1+\beta-\frac{\alpha_{1}}{1+\Gamma}\right) \alpha_{\lim }^{3}=(2+\beta)\left[\frac{1+\Gamma}{\alpha_{1}}\left(1+\frac{\beta^{4} I}{2 \Gamma^{4}}\right)-\alpha_{1}^{3}\right]-C,
$$

being $C$ the constant defined in (4.20) and $I$ the integral calculated in Appendix A. Conversely, if $\alpha_{\max }$ $<\alpha_{\lim }$ then the unloading process is entirely elastic.

A further decrease in the applied torque, namely for $\mu<\mu_{2}$ and thus $\alpha<\alpha_{2}$, then triggers the Martensite reorientation process starting from the boundary between the fully martensitic outer region and the intermediate transforming region, namely at $r=r_{f}$. From this circle, a new transforming region spreads out both inward and outward, where the positive variant Martensite is twisted into the negative one, according to eqn (2.6) for $\xi_{0}=\xi_{\max }$. This new region is delimited by the inner radius $r^{\prime}$ and the outer radius $r^{\prime \prime}$, where $r_{s} \leq r^{\prime} \leq r_{f} \leq r^{\prime \prime} \leq R$. Then, the shear stress at $r^{\prime}$ and $r^{\prime \prime}$ must coincide with the negative threshold stress $-\tau_{s}$ and it follows from elastic unloading starting from the shear stress $\tau_{\max }$ at the end of the loading process, namely

$$
\tau_{\max }\left(r^{\prime}\right)-\tau_{s} \frac{\alpha_{\max }-\alpha}{1+\Gamma \xi_{\max }\left(r^{\prime}\right)} \frac{r^{\prime}}{R}=-\tau_{s}, \quad \tau_{\max }\left(r^{\prime \prime}\right)-\tau_{s} \frac{\alpha_{\max }-\alpha}{1+\Gamma} \frac{r^{\prime \prime}}{R}=-\tau_{s},
$$

for $r_{s} \leq r^{\prime} \leq r_{f} \leq r^{\prime \prime} \leq R$. By introducing in (4.27) 1 the stress field $\tau_{\max }(r)$ at the end of the loading process given in (4.17) for $r_{s} \leq r \leq r_{f}$, one can find the angle of twist as a function of the radius $r^{\prime}$ :

$$
\alpha=\alpha_{\max }-\left[2+\beta \xi_{\max }\left(r^{\prime}\right)\right]\left[1+\Gamma \xi_{\max }\left(r^{\prime}\right)\right] \frac{R}{r^{\prime}}, \quad \text { for } r^{\prime} \leq r \leq r_{f} .
$$

Then, solving eqn (4.28) for $r^{\prime}$, by using eqn (4.18) for $\xi_{\max }\left(r^{\prime}\right)$ with $\alpha=\alpha_{\max }$, one can find the radius $r^{\prime}$ as a function of the normalized angle of twist $\alpha$

$$
\frac{r^{\prime}}{R}=\left(\frac{1+2 \delta}{\Gamma}-\frac{2}{\beta}\right) \frac{(\delta \beta-2 \Gamma) \alpha_{\max }-(1+\delta) \beta \alpha}{\left(\alpha_{\max }+\alpha\right)^{2}}\left\{1-\sqrt{1-\frac{2 \beta \Gamma\left(\alpha_{\max }+\alpha\right)^{2}}{\left[(\delta \beta-2 \Gamma) \alpha_{\max }-(1+\delta) \alpha \beta\right]^{2}}}\right\} .
$$

Similarly, by introducing in $(4.27)_{2}$ the stress field $\tau_{\max }(r)$ at the end of the loading process given in (4.17) for $r>r_{f}$, one can find the radius $r^{\prime \prime}$ as a function of $\alpha$

$$
\frac{r^{\prime \prime}}{R}=\frac{1}{\alpha}\left[\alpha_{1}-(1+\Gamma)(\beta+2)\right], \quad \text { for } r_{f} \leq r^{\prime \prime} \leq R .
$$

According to eqn (4.28), a material point at radius $r$ ranging between $r^{\prime}$ and $r_{f}$ starts experiencing 
Martensitic reorientation when $\alpha=\alpha_{r}$, where

$$
\alpha_{r}=\alpha_{\max }-\left[2+\beta \xi_{\max }(r)\right]\left[1+\Gamma \xi_{\max }(r)\right] \frac{R}{r}, \quad \text { for } r^{\prime} \leq r \leq r_{f},
$$

and thus from (4.28) and (4.31) it follows

$$
\alpha_{r}-\alpha=\left[2+\beta \xi_{\max }\left(r^{\prime}\right)\right]\left[1+\Gamma \xi_{\max }\left(r^{\prime}\right)\right] \frac{R}{r^{\prime}}-\left[2+\beta \xi_{\max }(r)\right]\left[1+\Gamma \xi_{\max }(r)\right] \frac{R}{r}, \text { for } r^{\prime} \leq r \leq r_{f} .
$$

Let us now consider a material point placed at radius $r$ ranging between $r_{f}$ and $r^{\prime \prime}$ which also starts experiencing Martensite reorientation when $\alpha=\alpha_{r}$. According to (4.30), $\alpha_{r}$ must obey the following condition also

$$
\alpha_{r}=\left[\alpha_{1}-(1+\Gamma)(\beta+2)\right] \frac{R}{r}, \quad \text { for } r_{f} \leq r \leq r^{\prime \prime},
$$

and thus from (4.30) and (4.33) it follows

$$
\alpha_{r}-\alpha=\left[\alpha_{1}-(1+\Gamma)(\beta+2)\right]\left(\frac{R}{r}-\frac{R}{r^{\prime \prime}}\right), \quad \text { for } r_{f} \leq r \leq r^{\prime \prime} .
$$

During the Martensite reorientation process, the following rate constitutive relation holds for a point at radius $r$, in agreement with eqns (2.3) and (4.3):

$$
\frac{r}{R} \dot{\alpha}=\left[1+\xi_{\text {tot }} \Gamma+\delta\left(1+\xi_{\max }\right)\right] \frac{\dot{\tau}}{\tau_{s}}, \quad \text { for } r^{\prime} \leq r \leq r^{\prime \prime},
$$

where relations (4.1) and (4.8) have been used. By introducing relation (2.7) for $\xi_{\text {tot }}$ with $\xi_{0}=\xi_{\max }$ in the rate constitutive equation (4.35) and integrating from the start of the Martensite reorientation at radius $r$, one gets

$$
\frac{r}{R}\left(\alpha-\alpha_{r}\right)=\frac{1}{\tau_{s}} \int_{-\tau_{s}}^{\tau}\left[1+\Gamma+\delta\left(1+\xi_{\max }\right)-\Gamma\left(1-\xi_{\max }\right) \frac{\tau+\tau_{f}}{\tau_{f}-\tau_{s}}\right] \mathrm{d} \tau, \text { for } r^{\prime} \leq r \leq r^{\prime \prime},
$$

being $-\tau_{s}, \alpha_{r}$ and $\xi_{\max }$ the shear stress, the normalized angle of twist and the Martensite volume fraction at the beginning of the Martensite reorientation at radius $r$, respectively. For $\xi_{\max }<1$, after calculation of the definite integral in (4.36), one obtains

$$
\frac{\tau^{2}}{\tau_{s}^{2}}+2\left[1-\beta \frac{1+\delta+(\delta-\Gamma) \xi_{\max }}{\Gamma\left(1-\xi_{\max }\right)}\right]\left(\frac{\tau}{\tau_{s}}+1\right)-1-\frac{2 \beta}{\Gamma} \frac{\alpha_{r}-\alpha}{1-\xi_{\max }} \frac{r}{R}=0, \quad \text { for } r^{\prime} \leq r \leq r_{f},
$$

where $\tau$ and $\alpha$ denote the shear stress and the normalized angle of twist during the Martensite reorientation process.

For $\xi_{\max }<1$, namely for $r^{\prime}<r<r_{f}$, eqn (4.37) can be solved for $\tau / \tau_{s}$ as

$$
\frac{\tau}{\tau_{s}}=-1-\beta \frac{1+\delta+(\delta+\Gamma) \xi_{\max }}{\Gamma\left(1-\xi_{\max }\right)}\left\{\sqrt{1+\frac{2 \Gamma\left(1-\xi_{\max }\right)\left(\alpha_{r}-\alpha\right)}{\beta\left[1+\delta+(\delta+\Gamma) \xi_{\max }\right]^{2}} \frac{r}{R}}-1\right\}, \quad \text { for } r^{\prime} \leq r \leq r_{f},
$$


where the term $\alpha_{r}-\alpha$ is given in (4.32), and $r^{\prime}$ is given in (4.29) as a function of $\alpha$.

For $\xi_{\max }=1$, namely for $r_{f} \leq r \leq r^{\prime \prime}$, eqn (4.36) provides instead

$$
\frac{\tau}{\tau_{s}}=-1-\frac{\alpha_{r}-\alpha}{1+\Gamma+2 \delta} \frac{r}{R}, \quad \text { for } r_{f} \leq r \leq r^{\prime \prime},
$$

where $r^{\prime \prime}$ is given in (4.30) as a function of $\alpha$, and $\alpha_{r}-\alpha$ is given in (4.34).

The other regions of the cross section, namely the inner part for $0 \leq r \leq r^{\prime}$ and the outer part for $r^{\prime \prime} \leq$ $r \leq R$, experience only elastic unloading from $\alpha_{\max }$ to $\alpha$, and thus the shear stress within this part is given by

$$
\frac{\tau}{\tau_{s}}=\frac{\tau_{\max }}{\tau_{s}}-\frac{\alpha_{\max }-\alpha}{1+\Gamma \xi_{\max }} \frac{r}{R}, \quad \text { for } 0 \leq r \leq r^{\prime} \text { and } r^{\prime \prime} \leq r \leq R .
$$

The corresponding distribution of effective Martensite across the regions where the negative Martensite variant is produced then follows from (2.6) for $\xi_{0}=\xi_{\max }$

$$
\xi=\xi_{\max }+\frac{1+\xi_{\max }}{\beta}\left(\frac{\tau}{\tau_{s}}+1\right), \quad \text { for } r^{\prime} \leq r \leq r^{\prime \prime},
$$

where the ratio $\tau / \tau_{s}$ is given by (4.38) for $r^{\prime} \leq r \leq r_{f}$, or by (4.39) for $r_{f} \leq r \leq r^{\prime \prime}$.

During unloading, the volume fraction of Martensite for $r<r^{\prime}$ and $r>r^{\prime \prime}$ does not change and is equal to $\xi_{\max }$, which follows from (4.18) for $\alpha=\alpha_{\max }$. Moreover, both the shear stress and the Martensite volume fraction display non-linear variations with the radius $r$ within the cross section, except for the inner austenitic region delimited by the radius $r_{s}$, where these variations are linear, according to eqns (4.40) and (4.41) for $\xi_{\max }=0$.

If the applied torque is decreased or applied with the opposite sign, the front of the start reorientation of the Martensite variant moves inward and outward, and it may reach the outer surface when $r^{\prime \prime}=R$, namely for $\alpha=\alpha_{3}$, where

$$
\alpha_{3}=\alpha_{1}-(1+\Gamma)(\beta+2), \quad \text { for } r_{f} \leq r^{\prime \prime} \leq R .
$$

according to (4.30). The shear stress and Martensite distributions during this stage are still given by eqns (4.38)-(4.41). Therefore, the introduction of the shear stress (4.38)-(4.40) in the balance condition (3.3) 1 yields the torque-twist relation for $\alpha_{3} \leq \alpha \leq \alpha_{2}$.

\subsection{Reversed loading unloading cycle}

If a negative torque is applied after the complete unloading of the bar and its magnitude is gradually increased, then the outer region made of positive Martensite variant disappears as the radius $r^{\prime \prime}$ 
provided by (3.24) 2 turns out to be larger than $R$, namely for $\alpha<\alpha_{3}$. The shear stress and Martensite distributions during this stage are given by eqns (4.38)-(4.41) for $r^{\prime \prime}>R$, and the corresponding torque-twist relation follows from the introduction of the shear stress fields in the balance condition $(3.3)_{1}$.

This stage of the reversed torsional loading ends when one of the following two conditions is first attained: the threshold stress $-\tau_{s}$ for the start of the reorientation process is reached at radius $r^{\prime}$, namely for $r^{\prime}=r_{s}$; or alternatively the threshold stress $-\tau_{f}$ for the finish of the reorientation process is reached at the outer surface, namely for $\tau(R)=-\tau_{f}$ or equivalently $r_{-f}=R$. According to (4.28), the former condition is attained for $\alpha=-\alpha \max$, being $\xi_{\max }\left(r_{s}\right)=0$ and $\alpha \max =R / r_{s}$. According to (4.39) and using relations (4.30), (4.34), and (4.12), the latter condition occurs for $\alpha=\alpha_{4}$, where

$$
\alpha_{4}=-\alpha_{1}-\Gamma(\beta+2) \text {. }
$$

If the magnitude of the negative torque is further increased, then a region fully made of the negative Martensite variant originates at the outer part of the cross section and propagates inwards with radius $r_{-f}$ defined by the condition $\tau\left(r_{-f}\right)=-\tau_{f}$, namely

$$
\frac{r_{-f}}{R}=-\frac{\alpha_{1}+(\beta+2) \Gamma}{\alpha},
$$

according to (4.33) and (4.39). In this case, the distributions of the shear stress and the effective Martensite volume fraction are defined by eqns (4.40) and (4.18) for $0 \leq r \leq r^{\prime}$, by (4.38) and (4.41) for $r^{\prime} \leq r \leq r_{f}$, and by (4.39) and (4.41) for $r_{f} \leq r \leq r_{-f}$, respectively, where the radii $r^{\prime}$ and $r^{\prime \prime}$ are given in (4.30) as functions of $\alpha$. Moreover, integration of eqn (2.3) in the outer fully martensitic region where $\xi=-1$, starting from the finish of martensitic transformation at $r$, by using (4.1) and (4.44), then gives

$$
\frac{\tau}{\tau_{s}}=-1-\beta+\frac{1}{1+\Gamma}\left[\alpha_{1}+(\beta+2) \Gamma+\alpha \frac{r}{R}\right], \quad \text { for } r_{-f} \leq r \leq R .
$$

The results obtained for equal shear moduli of the two phases are thus recovered for $\Gamma=0$. Note from eqn (4.44) that for $\Gamma>0$ and $\alpha=-\alpha_{\max }$, then $r^{\prime}=r_{s}$, but $r_{-f}$ does not coincide with $r_{f}$. Moreover, for $\Gamma>0$ the radial distributions of shear stress and Martensite at the end of the reversed loading process for $\alpha=-\alpha_{\max }$, which are given by eqns (4.18), (4.38)-(4.41) for $r^{\prime}=r_{s}$, are not exactly opposite to those obtained at the end of the first loading process for $\alpha=\alpha_{\max }$, as it instead occurs for equal shear moduli of the two phases, namely for $\Gamma=0$.

The introduction of the shear stress fields (4-38), (4.39), and (4.40) or (4.45) in the balance condition (3.3) $)_{1}$ then yields the torque-twist relation for the last stage of reversed torsional loading. 
Let $\tau_{\min }$ and $\xi_{\min }$ denote the radial distributions of shear stress and effective volume fraction of Martensite at the end of the reversed loading process for $\alpha_{\min }=-\alpha_{\max }$. They follow from (4.38)(4.41) and (4.45), by using (4.12), (4.31), and (4.33), as

$$
\frac{\tau_{\min }}{\tau_{s}}=\left\{\begin{array}{ll}
-\alpha_{\max } \frac{r}{R}, & \text { for } 0 \leq r \leq r_{s}, \\
-1-\beta \frac{1+\delta+(\delta+\Gamma) \xi_{\max }}{\Gamma\left(1-\xi_{\max }\right)}\left\{\sqrt{1+2 \Gamma\left(1-\xi_{\max }\right) \frac{2 \alpha_{\max } r / R-\left(2+\beta \xi_{\max }\right)\left(1+\Gamma \xi_{\max }\right)}{\beta\left[1+\delta+(\delta+\Gamma) \xi_{\max }\right]^{2}}}-1\right.
\end{array}\right\},
$$

and

$$
\xi_{\min }= \begin{cases}0, & \text { for } 0 \leq r \leq r_{s}, \\ \xi_{\max }+\frac{1+\xi_{\max }}{\beta}\left(\frac{\tau_{\min }}{\tau_{s}}+1\right), & \text { for } r_{s} \leq r \leq \min \left\{r_{-f, \min }, R\right\}, \\ -1, & \text { for } \max \left\{r_{-f, \min }, R\right\} \leq r \leq R,\end{cases}
$$

where

$$
r_{-f, \min }=\frac{\alpha_{1}+(\beta+2) \Gamma}{\alpha_{\max }} R .
$$

The shear stress during subsequent elastic unloading then follows from the integration of the rate constitutive equation (2.3) for $\dot{\xi}=0$, with $\xi=\xi_{\min }$, by using (4.1) for $\xi_{\text {tot }}=\xi_{\max }$

$$
\frac{\tau}{\tau_{s}}=\frac{\tau_{\min }}{\tau_{s}}+\frac{\alpha_{\text {max }}+\alpha}{1+\Gamma \xi_{\max }(r)} \frac{r}{R}, \quad \text { for } 0 \leq r \leq R .
$$

According to (3.3) $)_{1}$ and (4.49) the applied torque is

$$
\mu=\mu_{\min }+\left(\alpha_{\max }+\alpha\right) m .
$$

The complete unloading of the bar is attained for $\mu=0$. Correspondingly, the normalized twisting angle is

$$
\alpha_{\text {res }}^{\prime}=-\alpha_{\max }-\mu_{\min } / m \text {. }
$$

According to (4.49) the positive threshold stress $\tau_{s}$ is attained at $r=\min \left\{r_{-f, \min }, R\right\}$ for $\alpha=\alpha_{5}$ where 


$$
\alpha_{5}= \begin{cases}-\frac{\alpha_{1}-\beta-2}{\alpha_{1}+(\beta+2) \Gamma} \alpha_{\max }, & \text { if } r_{-f, \min }<R, \\ -\frac{(1+\Gamma)(\beta \Gamma-2-8 \delta-2 \beta \delta)+4 \delta \alpha_{\max }}{2(1+\Gamma+2 \delta)}, & \text { if } R \leq r_{-f, \min } .\end{cases}
$$

A further increase in the applied torque will cause the Martensite reorientation from the negative variant to the positive one starting from the circle at $r=\min \left\{r_{-f, \min }, R\right\}$.

\section{Results}

The constitutive parameters for NiTinol at temperature $T=298^{\circ} \mathrm{K}$ provided by Brinson (1993) are considered in the following. The values of the elastic shear moduli of Austenite and Martensite $G_{\mathrm{A}}$ and $G_{\mathrm{M}}$ and their mean value $G$, the Poisson coefficient $\nu$, the threshold shear stresses $\tau_{s}$ and $\tau_{f}$, and the maximum residual longitudinal strain $\varepsilon_{L}$ are reported in Table 1. If the same shear modulus equal to the mean value $G$ is assumed for both phases, then the constitutive parameters are $\beta=0.4575$ and $\delta=50.36$, whereas for different shear moduli the constitutive parameters are $\beta=0.4575, \delta=72.33$ and $\Gamma=1.548$.

The variations of the radii of start and finish martensitic transformation, $r_{s}$ and $r_{f}$, during the loading process with the normalized applied torque $\mu$ are plotted in Fig. 2, both for equal (dashed line) and different (solid line) shear moduli of the two phases. The variation of $r_{s}$ is almost similar for both models, thus implying that the dependence of the shear modulus on the Martensite content has limited influence on the size of the inner austenitic region. The size of the outer fully martensitic region, bounded inside by the radius $r_{f}$, is instead much larger if different shear moduli are considered for the two phases. As a result, the amount of transformed Martensite within the cross section is also larger. The variations with the constitutive parameter $\delta$ of the normalized torque $\mu_{1}$ defined in (3.11), corresponding to the achievement of the threshold shear stress $\tau_{f}$ at the outer surface of the bar, are plotted in Fig. $3 a$, for some values of the ratio $\beta$ and for equal shear moduli of the two phases. The normalized torque $\mu_{1}$ turns out to be almost independent of $\delta$ for large values of $\delta$ and it grows up as the threshold stress $\tau_{f}$ is increased, namely with $\beta$. The variation with $\delta$ of the limit torque $\mu_{\lim }$ satisfying eqn (3.22), which causes Martensite reorientation before the complete unloading, are plotted in Fig. $3 b$ for equal shear moduli of the two phases. The limit torque $\mu_{\text {lim }}$ is found here to decrease monotonically with $\delta$. The variation of $\mu_{\lim }$ with $\beta$ is instead more complex: it decreases for small values of $\delta$ and it grows up for large values of $\delta$.

Similar variations are plotted in Fig $4 a$ and $4 b$ for different shear moduli of the two phases, precisely 
for the ratio $G_{\mathrm{A}} / G_{\mathrm{M}}$ equal 2 and 3, respectively. The trend is similar to that observed for equal shear moduli. Moreover, the limit torque $\mu_{\mathrm{lim}}$ increases with the ratio $G_{\mathrm{A}} / G_{\mathrm{M}}$ and thus with $\Gamma$, whereas the normalized torque $\mu_{1}$ is almost independent of this ratio.

In the following, the radial distributions of shear stress and Martensite volume fraction during the loading cycle are plotted and discussed, both for equal and different shear moduli of the two phases, in order to compare the two analytical models developed in Sections 3 and 4.

The radial distributions of the shear stress and effective volume fraction of Martensite at the end of the loading process for $\alpha=\alpha_{\max }$, at the end of elastic unloading for $\alpha=\alpha_{2}$, after complete unloading for $\alpha=\alpha_{\text {res, }}$, and during the reverse loading process for $\alpha=\alpha_{3}, \alpha=-\alpha_{1}$ and $\alpha=-\alpha_{\max }$ are plotted in Fig. 5 for $\mu_{\max }=2$, and in Fig. 6 for $\mu_{\max }=4$, under the assumption of equal shear moduli of the two phases. In this case, the limit torque satisfying eqn (3.22) is $\mu_{\mathrm{lim}}=2.857$. During the first loading process, the radial distribution of the shear stress is linear across each region and it considerably increases in the outer fully martensitic region, so that the yield condition of the SMA could be first attained at the outer surface, as expected. A similar trend was observed by Mirzaeifar et al. (2010) and Viet et al. (2019) in their analytical investigations. For $\mu_{\max }=2<\mu_{\lim }$ the unloading process is entirely elastic and after complete unloading a wide annular region is subject to negative shear stress (black line in Fig. 5a), whose magnitude however is not sufficient for triggering the Martensite reorientation process. For a maximum applied torque $\mu$ larger than $\mu_{\text {lim }}\left(\right.$ e.g. for $\left.\mu_{\max }=4\right)$ Martensite reorientation occurs during the unloading process at first in an annular region surrounding the circle of radius $r_{f}$ and spreading inwards and outwards as the applied torque is decreased. Moreover, its size increases with the maximum applied torque at the end of the loading process. In this region, the shear stress $\tau$ at the end of elastic unloading displays a weak and limited nonlinear variation with the radius $r$, according to (3.30), being actually almost constant and a bit more negative than $-\tau_{s}$ (black line in Fig. 6a).

For equal shear moduli, the radial distribution of the effective Martensite volume fraction $\xi$ across the transforming region during the loading process turns out to be linear from 0 to 1 . For $\mu_{\max }>\mu_{\lim }$ at the end of the unloading process, the Martensite reorientation occurred in the annular region delimited by the radii $r^{\prime}$ and $r^{\prime \prime}$ (black line in Fig. 6b). The amount of Martensite reoriented during the unloading process increases with the maximum applied torque $\mu_{\max }$.

During the subsequent reversed torsional loading, the volume fraction of negative variant Martensite increases and the effective volume fraction of Martensite $\xi$ decreases correspondingly. However, the total amount of Martensite does not change $\left(\xi_{\text {tot }}=\xi_{\max }\right)$.

As the magnitude of the applied negative torque is increased, the annular region where Martensite 
reorientation occurs, namely where $\tau<-\tau_{s}$, at first reaches the outer surface of the cross section for $\alpha=\alpha_{3}$ and then it tends to recover the entire region where the martensitic transformation occurred during the loading process, namely the annular region ranging from the inner radius $r_{s}$ to the outer radius $R$. Note that for $\alpha=0$, namely when the twist is completely removed from the rod, a limited amount of positively twisted Martensite is still present within the cross section (green lines in Figs. $5 b$ and $6 b$ ). Finally, for $\alpha=-\alpha_{\max }$ the shear stress and effective Martensite distributions are exactly opposite to those observed at the end of the loading process for $\alpha=\alpha_{\max }$, and thus the negative variant Martensite has completely replaced the positive one within the cross section.

The radial distributions of the shear stress for different shear moduli of the two phases are plotted in Fig. $7 a$ for $\mu_{\max }=2$ and in Fig. $8 a$ for $\mu_{\max }=4$ at various stages of the process, namely at the end of direct loading for $\alpha=\alpha_{\max }$ (blue line), at the end of elastic unloading for $\alpha=\alpha_{2}$ (red line), after complete unloading or $\alpha=\alpha_{\text {res }}$ (black line), and during reverse loading for $\alpha=\alpha_{3}, \alpha=0, \alpha=-\alpha_{1}$ and $\alpha=-\alpha_{\max }$. These variations are considerably different from those plotted in Figs. $5 a$ and $6 a$ for equal shear moduli. Indeed, the maximum shear stress attained at the outer surface at the end of the loading process now is a bit lower and the purely martensitic outer region is a bit larger than those observed for equal shear moduli. Moreover, for different shear moduli the radial distribution of the shear stress is weakly non-linear in the transforming regions during first loading and clearly nonlinear during the unloading and negative reloading processes. Finally, the radial distributions of the shear stress and effective Martensite volume fraction at the end of the negative reloading process for $\alpha=$ $-\alpha_{\max }$ (blue line) are not exactly opposite to those obtained for $\alpha=\alpha_{\max }$, as it occurs for equal shear moduli. The magnitude of the shear stress is instead smaller than that observed at the end of the first loading process and thus the positive variant Martensite is still present, due to the assumption of different shear moduli. The corresponding radial variations of the effective Martensite volume fraction $\xi=\xi^{+}-\xi^{-}$are plotted in Fig. $7 b$ for $\mu_{\max }=2$ and in Fig. $8 b$ for $\mu_{\max }=4$, according to eqns (4.18) and (4.41). These plots show that the radial distribution of $\xi$ across the transforming region is non-linear, also during the first loading process, although it is very close to a linear trend.

The radial distributions of the residual shear stress upon complete unloading after first loading for $\alpha=\alpha_{\text {res }}$ are plotted in Fig. $9 a$ for some values of $\mu_{\max }$ and for different shear moduli of the two phases. Note that the rate behavior of the section is still elastic for $\mu_{\max }<\mu_{\text {lim. }}$. For $\mu_{\max }>\mu_{\text {lim }}$ the minimum residual shear stress displays a plateau a little bit lower than $-\tau_{s}$, which extends within the cross section as the maximum applied torque is increased. The maximum residual shear stress is attained at the outer surface and it increases remarkably with $\mu_{\max }$. Similar variation are plotted in Fig. $9 b$ for the residual shear stress upon complete unloading subsequent to negative reloading, namely for $\alpha=$ 
$\alpha^{\prime}$ res. The minimum residual shear stress is attained at the outer surface and its magnitude increases remarkably with $\mu_{\max }$. Note that for the three values of $\mu_{\max }$ considered in Fig. $9 b$ the complete unloading of the section occurs under rate elastic behavior, since the maximum residual shear stress is lower than the threshold stress $\tau_{s}$.

The variations of the angles of twist at the end of the unloading process $\alpha_{\text {res }}$ and at the end of the elastic unloading stage $\alpha_{2}$ with the maximum angle of twist $\alpha_{\max }$ are plotted in Fig.10a for equal shear moduli of the two phases and in Fig. $10 \mathrm{~b}$ for different shear moduli. These plots also show the variation of the angles of twist $\alpha_{1}$ and $\alpha_{3}$ (dashed lines), corresponding respectively to start of the transformation into positive Martensite variant at the outer surface during first loading and to start of the Martensite reorientation at the outer surface during unloading or reverse loading. As the angle of twist $\alpha$ is decreased during the unloading process, the complete unloading occurs under elastic unloading for small values of $\alpha_{\max }$, whereas it occurs after elastic unloading for large values of $\alpha_{\max }$. The normalized variations of the radii $r_{s}, r^{\prime}, r_{f}, r^{\prime \prime}$, and $r_{-f}$ with the angle of twist during the unloading and reverse loading processes, both for $\mu_{\max }=2$ and $\mu_{\max }=4$, are plotted in Fig.11 for equal shear moduli of the two phases and in Fig. 12 for different shear moduli. It can be observed that the radius $r^{\prime}$ (red line) tends to $r_{s}$ (dotted line) as the angle of twist tends to $-\alpha_{\max }$, namely as the reverse loading is increased. Moreover, for equal shear moduli a fully negative martensitic region takes place at the outer part of the cross section for $r \geq r_{-f}$ (green line) and extends inwards, till it reaches the radius $r_{f}$ (dashed line) for $\alpha=-\alpha_{\max }$, thus replacing the fully positive martensitic region transformed during the loading process. As a difference, for different shear moduli the fully negative martensitic region does not appear for $\alpha=-\alpha_{\max }$ if $\mu_{\max }=2$, but it occurs for $\mu_{\max }=4$. In this case, however, it does not replace completely the fully positive martensitic region for $\alpha=-\alpha_{\max }$.

The closed form relations between the normalized applied torque $\mu$ and angle of twist $\alpha$ for equal shear moduli under cyclic torsional loading of alternating sign, defined by eqns (3.4), (3.10), (3.15), (3.20), and (3.37), are plotted in Fig. $13 a$ for $\mu_{\max }=2$, 3, and 4. If the Martensite reorientation process is triggered during the unloading process then the residual shear strain decreases a little bit with respect to a pure elastic unloading. Moreover, the minimum torque and twist at the end of reversed loading are exactly opposite to the maximum ones. As already observed, the distributions of shear stress and Martensite variants at the end of the first loading process and at the end of the reversed loading process are indeed equal but opposite in sign Therefore, the perfectly opposite results obtained here for $\Gamma=0$ under direct and reverse torsional loading hold also for multiple loading cycles of alternating sign. 
The torque-twist relations for different shear moduli of the two phases are plotted in Fig. 13b. The analytical relations for the first loading and subsequent elastic unloading stages are given in (4.4), (4.13), (4.19) and (4.23). The torque-twist relations for the subsequent stages of the reversed torsional loading follow from the introduction of the shear stress (4.38)-(4.40), and (4.45) in the integral in eqn (3.3) $)_{1}$. In this case, the slope of the last part of the loading process as well as that of the elastic unloading process is clearly smaller than in Fig. $13 a$, as due to the lower shear modulus of Martensite considered here. This last occurrence also makes the residual angle of twist after complete unloading a little bit smaller than that obtained for equal shear moduli. Therefore, the simplifying assumption of equal shear moduli of the two phases turns out in simpler analytical expressions for the shear stress and Martensite distributions, but it actually overestimates the SM effect.

Moreover, the area enclosed by each cycle in Fig. $13 b$ is clearly larger than that observed for equal shear moduli in Fig. $13 a$, due to the larger twist exhibited by the refined model, thus denoting a higher energy dissipation of that model under the same maximum applied torque.

Note also that the response under reversed torsional loading is not perfectly opposite to the response under direct loading, namely the stress field and the Martensite distribution at the end of reversed loading is not exactly opposite to those observed at the end of first loading, although a symmetric constitutive behavior has been assumed. The difference is due to the different shear moduli of the two phases. Indeed, the response becomes perfectly opposite for $\Gamma=0$, namely for $G_{\mathrm{A}}=G_{\mathrm{M}}$, as observed in Fig. $13 a$.

Here, the analysis has been developed only for the first cycle of loading-unloading and reverse loading-unloading. In theory, it can be extended to subsequent cycles. However, the analytical difficulties remarkably increase for different shear moduli of the two phases. On the contrary, if the same shear modulus is assumed for both phases, namely for $\Gamma=0$, then the response during repeated loading cycles of the same amplitude does not change. In this case, the response observed in the first cycle is exactly reiterated in subsequent cycles of the same amplitude, as in Fig. $13 a$.

In order to validate the present analysis, a comparison with some analytical results available in the technical literature is also provided in Fig. 14. In particular, the torque-twist relations for a circular cross section of radius $R=25 \mathrm{~mm}$ during the loading process at three different temperatures provided in Figs. 9 and 10 of Mirzaeifar et al. (2010) for their Material I (dotted lines) are compared with the present solution for different shear moduli (solid lines). The constitutive parameters of Material I at three different temperatures are given in Tab. 2. The curves are very close, except for the last stage of the loading process at the higher temperature, being the slope of the present solution a bit higher than that predicted by Mirzaeifar et al. (2010). Unfortunately, no results are available in the technical literature for the Martensite reorientation process under reversed torsional loading at temperature 
lower than $A_{s}$, which can be compared with the present ones. Experimental results of complex loading and unloading torsional cycles under isothermal conditions were conducted by Rao and Srinivasa (2013) and Rao et al. (2014), but only on the SE response of SMA wires.

The torque-twist relation here provided for the loading unloading process can be straight exploited for modeling the response of SMA helical springs with a small helix angle and a mean coil radius $R_{m}$ much greater than the cross section radius $R$. In this case, the axial force $F$ acting on the spring and the corresponding axial displacement $u$ are given by (Mirzaeifar et al., 2011)

$$
F=\frac{M}{R_{m}}=\mu \frac{M_{s}}{R_{m}}, \quad u=2 \pi N R_{m}^{2} \theta=2 \pi N \frac{\tau_{s} R_{m}^{2}}{G_{A} R} \alpha,
$$

in terms of $\mu$ and $\alpha$, where $N$ is the number of coils. Therefore, if the curvature and pitch effects can be neglected, the relation between $\mu$ and $\alpha$ proposed here also describes the force-displacement relation for a helical spring.

A comparison between the analytical results provided by the present formulation for different shear moduli of the two phases and the numerical and experimental findings available in the literature for a SMA spring under a loading-unloading cycle at temperature allowing for the SM effect is given in Fig. 15. In particular, the relation between tensile force and displacement for a SMA spring with $N=$ $5, R_{m}=4.5 \mathrm{~mm}$ and $R=0.75 \mathrm{~mm}$, whose constitutive parameters at room temperature $T=17^{\circ} \mathrm{C}$ are reported in Tab. 3, is compared in Fig. 15a with the corresponding numerical results plotted in Fig. $6 c$ of Hasemi and Kadkhodaei (2018) under the hypothesis of small deformation. A reasonable agreement can be observed between the results of the two formulations, although the simplifying assumption of a linear relation between Martensite volume fraction and shear stress made in the present work underestimates the applied force $F$ at the beginning of the martensitic transformation and overestimated it at the end of the transformation. However, the energy dissipated during the full loading-unloading cycle is well approximated by the present approach.

Moreover, the force-displacement relations for the compressive loading-unloading SM effect response of a SMA spring with $N=5, R_{m}=4.75 \mathrm{~mm}$ and $R=0.75 \mathrm{~mm}$ at $20^{\circ} \mathrm{C}$ and $21.5^{\circ} \mathrm{C}$, whose constitutive parameters are reported in Tab. 4, are compared in Fig. $15 b$ with the experimental results provided in Fig. 9 of Heidari et al. (2016). In this case the spring is unloaded much before the finish of martensitic transformation, and thus the present approach provides a lower value of the applied force $F$ with respect to the experimental results. Obviously, the more realistic adoption of a non-linear evolution law for the Martensite volume fraction with the shear stress would provide results closer to the experimental ones, but it would not allow for obtaining a full analytical formulation.

The same framework developed here for simulating the Martensite reorientation can be employed 
also for modeling the reverse austenitic transformation, which occurs during the unloading process at temperatures higher than $A_{s}$.

Moreover, the model can be easily extended by taking into consideration asymmetric behavior of the SMA, which has been observed by Poorasadion et al. (2013) and Fahimi et al. (2019). In this case, different magnitudes must be assumed for the positive and negative threshold shear stresses. In this case the equations would result even more complex, though the procedure does not substantially change. The model can also be straight improved for simulating the torsional behavior of a hollow circular section and, thus, the performance of hollow helical SMA springs, which can be used for innovative applications as shape memory actuators with increased dynamic bandwidth, energetic efficiency and stroke (Spinella and Dragoni, 2010, Spinella et al., 2010).

\section{Conclusions}

Two simple 1D constitutive models are used for the macroscopic description of the torsional response of a circular SMA bar at constant temperature, in order to simulate the SM effect under cyclic loading of opposite sign. The basic model assumes the same shear modulus for both austenitic and martensitic phases, whereas the more complex model adopts the Reuss scheme for the elastic response of a composite of two phases with different elastic moduli. Both models include two opposite Martensite variants, whose volumetric fractions are assumed to evolve linearly with the shear stress, according to its sign, so that the piecewise-linear rate constitutive model can be integrated analytically within each step. These simplifying assumptions allow us to obtain a closed form solution for the radial distributions of shear stress and Martensite fractions during the first direct and reversed cyclic processes. The analytical or numerical integration of the shear stress within the cross section then yields the corresponding relation between the applied torque and the angle of twist.

It is shown that the simplifying assumption of equal shear moduli of the two phases turns out in simpler analytical expressions for the shear stress, Martensite distributions, and torque-twist relation, but it underestimates the hysteretic response of SMA bar under cyclic torsional loading. Indeed, the area enclosed by each loop for different shear moduli is larger than that observed for equal shear moduli under the same maximum applied torque, due to the larger twist exhibited by the model with two different shear moduli, thus denoting a higher energy dissipation capability of that model. Since the more complex model recovers the simpler one as $\Gamma$ tends to 0 , then the former can be used in any case if there is a need for more accurate results.

The assumption of different shear moduli of the two phases is also responsible of an asymmetric 
response under reversed torque with respect to the first loading process. If the two shear moduli are equal, namely $\Gamma=0$, then the torque-twist relations under direct and reversed loading-unloading cycles of the same amplitude are indeed perfectly opposite each other.

The analytical results provided here are considerably useful for the accurate design of innovative mechanical devices such as dampers, stiffeners and actuators realized by means of SMA helical springs. They can be efficiently used also for validating the accuracy of numerical methods usually employed in the complex modeling of cycling behavior of SMA dissipating devices. We caution the reader that, due to the number of factors that may influence the thermomechanical and hysteretic behavior of SMA dampers, it is suggested to verify their complex response with further experimentation.

One of the reviewers criticized the assumption of homogeneous deformation under pure torsional loading. As mentioned in the Introduction, there exists experimental evidence that the deformation in SMAs is inhomogeneous at the microscale. However, we provide here an analytical description of the SMA response at the macroscale, by using a simple and widely adopted phenomenological model of the SMA constitutive behavior. A more accurate and detailed micromechanical investigation would necessarily require the adoption of numerical strategies for simulating the SMA hysteretic response, also for simple structural components.

\section{Acknowledgements}

Support from the Italian "Gruppo Nazionale di Fisica Matematica" INdAM-GNFM is gratefully acknowledged.

\section{References}

Andani, M.T., Alipour, A., Eshghinejad, A., Elahinia, M., 2013. Modifying the torque-angle behavior of rotary shape memory alloy actuators through axial loading: A semi-analytical study of combined tension-torsion behavior. J. Intell. Mater. Syst. Struct. 24(12) 1524-1535.

Brinson, L.C., 1993. One-dimensional constitutive behavior of shape memory alloys: thermomechanical derivation with non-constant material functions and redefined Martensite internal variable. J. Intell. Mater. Syst. Struct. 4(2), 229-242.

Brinson, L.C., Huang, M.S., 1996. Simplifications and comparisons of shape memory alloy constitutive models. J. Intell. Mater. Syst. Struct. 7, 108-114. 
Bronstein, E., Faran, E., Shilo, D., 2019. Analysis of austenite-martensite phase boundary and twinned microstructure in shape memory alloys: The role of twinning disconnections. Acta Materialia, 164, 520-529.

Chapman, C., Eshghinejad, A., Elahinia, M., 2011. Torsional behavior of NiTi wires and tubes: Modeling and experimentation. J. Intell. Mater. Syst. Struct. 22, 1239-1248.

Chen, X., Peng, X., Chen, B., Han, J., Zeng, Z., Hu, N., 2014. Experimental investigation to thermalmechanical behavior of Ni47Ti44Nb9 SMA under pure tension and pure torsion. J. Alloys Compounds, 610, 151-160.

DesRoche, R., and Smith, B., 2004. Shape memory alloys in seismic resistant design and retrofit: a critical review of their potential and limitations. J. Earthquake Engng. 8, 415-429.

Dolce, M., Cardone, D., Marnetto, R., 2000. Implementation and testing of passive control devices based on shape memory alloys. Earthquake Engng. Struct. Dyn. 2000; 29, 945-968.

Dolce, M., Cardone, D., 2001. Mechanical behavior of shape memory alloy for seismic applications 1. Martensite and Austenite NiTi bars subject to torsion. Int. J. Mech. Sci. 43, 2631-2656.

Fang, C., Wang, W. 2020. Shape memory alloys for seismic resilience. Springer. Singapore.

Frost, M., Sedlák, P., Kadeřávek, L., Heller, L., Šittner, P., 2016. Modeling of mechanical response of NiTi shape memory alloy subjected to combined thermal and non-proportional mechanical loading: a case study on helical spring actuator. J. Intell. Mater. Syst. Struct., 27(14), 1927-1938.

Govindjee, S., Kasper, E.R., 1997. A shape memory alloy model for Uranium-Niobium accounting for plasticity. J. Intell. Mater. Syst. Struct. 8, 815-823.

Govindjee, S., Kasper, E.R., 1999. Computational aspects of one-dimensional shape memory alloy modeling with phase diagrams. Comput. Methods Appl. Mech. Eng. 171, 309-326.

Fahimi, P., Zakerzadeh, M. R., Baghani, M., 2019. A combined experimental and numerical study on shape memory alloy rods under torsion. J. Intell. Mater. Syst. Struct. 30(15), 2222-2233.

Hashemi, Y. M., Kadkhodaei, M., 2018. The Effects of geometric parameters under small and large deformations on dissipative performance of shape memory alloy helical springs. J. Stress Analysis 3(1), 69-79.

Hashemi, Y. M., Kadkhodaei, M., Mohammadzadeh M. R., 2019. Fatigue analysis of shape memory alloy helical springs. Int. J. Mech. Sci. 161-162, 105059.

Heidari, B., Kadkhodaei, M., Barati, M., Karimzadeh, F., 2016. Fabrication and modeling of shape memory alloy springs. Smart Mater. Struct. 25(12), 125003.

Liang, C., Rogers, C. A., 1990. One-dimensional thermomechanical constitutive relations for shape memory materials. J. Intell. Mater. Syst. Struct. 1(2), 207-234. 
Lim T.J., McDowell, D. L., 1999, Mechanical behavior of an Ni-Ti shape memory alloy under axialtorsional proportional and nonproportional loading. J. Eng. Mater. Technol. 121, 9-18.

Mahtabi, M. J., Shamsaei, N., Mitchell, M. R., 2015. Fatigue of Nitinol: The state-of-the-art and ongoing challenges. J. Mech. Behavior Biomed. Mater., 50, 228-254.

Marfia, S., Rizzoni, R., 2013. One-dimensional constitutive SMA model with two Martensite variants: Analytical and numerical solutions. Eur. J. Mech. A/Solids 40, 166-185.

Mirzaeifar, R, DesRoches, R., Yavari, A., 2010. Exact solutions for pure torsion of shape memory alloy circular bars. Mech. Mater. 420(8), 797-806.

Mirzaeifar, R., DesRoches, R., Yavari, A., 2011. A combined analytical, numerical, and experimental study of shape-memory-alloy helical springs. Int. J. Solids Struct. 48, 611-624.

Mirzaeifar, R., DesRoches, R., Yavari, A., Gall, K., 2012. Coupled thermo-mechanic analysis of shape memory alloy circular bars in pure torsion. Int. J. Non-Linear Mech. 47, 118-128.

Mohammadzadeh, M.R., Kadkhodaei, M., Barati, M., Chirani, S.A., Saint-Sulpice, L., 2019. Modeling of torsion fatigue in shape memory alloys. J. Intell. Mater. Syst. Struct. 30(20), 31463162.

Ozbulut, O.E., Hurlebaus, S., DesRoches, R., 2011. Seismic response control using shape memory alloys: a review. J. Intell. Mater. Syst. Struct. 22(14), 1531-1549.

Panoskaltsis, V.P., Bahuguna, S., Soldatos, D., 2004. On the thermomechanical modelling of shape memory alloys. Int. J. Nonlin. Mech. 39(5), 709-722.

Poorasadion S, Arghavani J, Naghdabadi R, et al. (2013) An improvement on the Brinson model for shape memory alloys with application to two-dimensional beam element. J. Intell. Mater. Syst. Struct. 25(15): 1905-1920.

Peng, X., Pi, W., Fan, J. (2008). A microstructure-based constitutive model for the pseudoelastic behavior of NiTi SMAs. Int. J. Plasticity, 24(6), 966-990.

Radi, E., 2018. Evolution of multiple Martensite variants in a SMA thick walled cylinder under internal pressure. Int. J. Solids Struct. 155, 15-35.

Radi E., 2021. Analytical modelling of the shape memory effect in SMA beams with rectangular cross section under reversed pure bending. J. Intell. Mater. Syst. Struct. https://doi.org/10.1177/1045389X20988789.

Rao, A., Srinivasa, A.R. 2013. A two species thermodynamic Preisach model for the torsional response of shape memory alloy wires and springs under superelastic conditions. Int. J. Solids Struct. 50, 887-898.

Rao. A., Ruimi, A., Srinivasa, A.R., 2014. Internal loops in superelastic shape memory alloy wires under torsion - Experiments and simulations/predictions. Int. J. Solids Struct. 51, 4554-4571. 
Rao A., Srinivasa A.R., Reddy J.N., 2015. Case studies in the preliminary design of SMA actuators. In: Design of Shape Memory Alloy (SMA) Actuators. Springer Briefs in Applied Sciences and Technology. Springer, Cham.

Reedlunn, B., LePage, W. S., Daly, S. H., Shaw, J. A. (2020). Axial-torsion behavior of superelastic tubes: Part I, proportional isothermal experiments. Int. J. Solids Struct. 199, 1-35.

Speicher, M., Hodgson, D.E., DesRoche, R., Leon, R.T., 2009. Shape memory alloy tension/ compression device for seismic retrofit of buildings. J. Mater. Perform. 18, 746-753.

Spinella, I., Dragoni, E., 2010. Analysis and design of hollow helical springs for shape memory actuators J. Intell. Mater. Syst. Struct. 21, 185-199.

Spinella, I., Dragoni, E., Stortiero, F., 2010. Modeling, prototyping, and testing of helical shape memory compression springs with hollow cross section. J. Mech. Des. 132, 061008.

Song, G., Ma, N., Li H.-N., 2006. Applications of shape memory alloys in civil structures. Engng. Struct. 28, 1266-1274.

Sun, Q. P., Li, Z. Q., (2002). Phase transformation in superelastic NiTi polycrystalline micro-tubes under tension and torsion - from localization to homogeneous deformation. Int. J. Solids Struct., 39(13-14), 3797-3809.

Torra, V., Isalgue, A., Lovey, F. C., Sade, M., 2015. Shape memory alloys as an effective tool to damp oscillations. J. Therm. Analysis Calorimetry, 119(3), 1475-1533.

Viet, N.V., Zaki, W., Umer, R., Cissé, C., 2019. Analytical model for the torsional response of superelastic shape memory alloy circular sections subjected to a loading-unloading cycle. Int. J. Solids Struct. 156-157, 49-60.

Viet, N.V., Zaki, W., Umer, R., Xu, Y., 2020. Mathematical model for superelastic shape memory alloy springs with large spring index. Int. J. Solids Struct. 185-186, 159-169.

Wilkes, K.E., Liaw, P.K., 2000. The fatigue behavior of shape-memory alloys. JOM 52, 45-51.

Wilson, J.C., Wesolowsky M.J. 2005. Shape memory alloys for seismic response modification: a state-of-the-art review. Earthquake Spectra 21(2), 569-601.

Zaki, W., 2012. An efficient implementation for a model of Martensite reorientation in martensitic shape memory alloys under multiaxial nonproportional loading. Int. J. Plast. 37, 72-94.

\section{Appendix A}

The integral in the definition of $m$ in eqn (4.24) can be calculated as 


$$
\begin{aligned}
I=\int_{1}^{1+\Gamma}\left[(t+\delta)^{2}-\right. & p]^{3}\left(1+\frac{\delta}{t}\right) d t=\frac{1}{7}\left[(1+\Gamma)^{7}-1\right]+\frac{7}{6}\left[(1+\Gamma)^{6}-1\right] \delta+\frac{3}{5}\left[(1+\Gamma)^{5}-1\right]\left(7 \delta^{2}-p\right)+ \\
& +\frac{5}{4}\left[(1+\Gamma)^{4}-1\right] \delta\left(7 \delta^{2}-3 p\right)+\frac{1}{3}\left[(1+\Gamma)^{3}-1\right]\left(35 \delta^{4}-30 p \delta^{2}+3 p^{2}\right) \\
& +\frac{3}{2}\left[(1+\Gamma)^{2}-1\right] \delta\left(7 \delta^{2}-3 p\right)\left(\delta^{2}-p\right)+\Gamma\left(7 \delta^{2}-p\right)\left(\delta^{2}-p\right)^{2}+\delta\left(\delta^{2}-p\right)^{3} \ln (1+\Gamma),
\end{aligned}
$$

where

$$
p=(1+\delta)^{2}-2 \frac{\Gamma}{\beta} .
$$

\section{TABLES}

\begin{tabular}{|c|c|c|c|c|c|c|c|}
\hline $\begin{array}{c}T \\
{\left[{ }^{\circ} \mathrm{K}\right]}\end{array}$ & $\begin{array}{c}G_{\mathrm{A}} \\
{[G P a]}\end{array}$ & $\begin{array}{c}G_{\mathrm{M}} \\
{[G P a]}\end{array}$ & $\begin{array}{c}G \\
{[G P a]}\end{array}$ & $v$ & $\begin{array}{c}\tau_{s} \\
{[M P a]}\end{array}$ & $\begin{array}{c}\tau_{f} \\
{[M P a]}\end{array}$ & $\varepsilon_{L}$ \\
\hline 298 & 25.2 & 9.9 & 17.5 & 0.33 & 88 & 129 & 0.067 \\
\hline
\end{tabular}

Table 1. Constitutive parameters for Brinson SMA materials.

\begin{tabular}{|c|c|c|c|c|c|c|}
\hline $\begin{array}{c}T \\
{\left[{ }^{\circ} \mathrm{K}\right]}\end{array}$ & $\begin{array}{c}G_{\mathrm{A}} \\
{[G P a]}\end{array}$ & $\begin{array}{c}G_{\mathrm{M}} \\
{[G P a]}\end{array}$ & $v$ & $\begin{array}{c}\tau_{s} \\
{[M P a]}\end{array}$ & $\begin{array}{c}\tau_{f} \\
{[M P a]}\end{array}$ & $\varepsilon_{L}$ \\
\hline 305 & 26.92 & 11.54 & 0.3 & 55 & 120 & 0.05 \\
\hline 315 & 26.92 & 11.54 & 0.3 & 88 & 153 & 0.05 \\
\hline 325 & 26.92 & 11.54 & 0.3 & 120 & 185 & 0.05 \\
\hline
\end{tabular}

Table 2. Constitutive parameters for Material I at three different temperatures, from Mirzaeifar et al. (2010). 


\begin{tabular}{|c|c|c|c|c|c|}
\hline $\begin{array}{c}T \\
{\left[{ }^{\circ} \mathrm{K}\right]}\end{array}$ & $\begin{array}{c}G_{\mathrm{A}} \\
{[G P a]}\end{array}$ & $\begin{array}{c}G_{\mathrm{M}} \\
{[G P a]}\end{array}$ & $\begin{array}{c}\tau_{s} \\
{[M P a]}\end{array}$ & $\begin{array}{c}\tau_{f} \\
{[M P a]}\end{array}$ & $\varepsilon_{L}$ \\
\hline 290 & 10.8 & 9.21 & 3.9 & 68 & 0.069 \\
\hline
\end{tabular}

Table 3. Constitutive parameters for SMA spring at $17^{\circ} \mathrm{C}$ from Hashemi and Kadkhodaei (2018).

\begin{tabular}{|c|c|c|c|c|c|}
\hline $\begin{array}{c}T \\
{\left[{ }^{\circ} \mathrm{K}\right]}\end{array}$ & $\begin{array}{c}G_{\mathrm{A}} \\
{[G P a]}\end{array}$ & $\begin{array}{c}G_{\mathrm{M}} \\
{[G P a]}\end{array}$ & $\begin{array}{c}\tau_{s} \\
{[M P a]}\end{array}$ & $\begin{array}{c}\tau_{f} \\
{[M P a]}\end{array}$ & $\varepsilon_{L}$ \\
\hline 293.15 & 10.8 & 9.2 & 28.7 & 140.4 & 0.069 \\
\hline 294.65 & 10.8 & 9.2 & 39.6 & 151.3 & 0.069 \\
\hline
\end{tabular}

Table 4. Constitutive parameters for SMA spring at two different temperatures, from Heidari et al. (2016). 